\title{
Agent-Based Computational Epidemiological Modeling
}

\author{
Keith R. Bissett ${ }^{1}$, Jose Cadena ${ }^{2}$, Maleq Khan ${ }^{3}$ and Chris J. Kuhlman ${ }^{4}$
}

\begin{abstract}
The study of epidemics is useful for not only understanding outbreaks and trying to limit their adverse effects, but also because epidemics are related to social phenomena such as government instability, crime, poverty, and inequality. One approach for studying epidemics is to simulate their spread through populations. In this work, we describe an integrated multi-dimensional approach to epidemic simulation, which encompasses: (1) a theoretical framework for simulation and analysis; (2) synthetic population (digital twin) generation; (3) (social contact) network construction methods from synthetic populations, (4) stylized network construction methods; and (5) simulation of the evolution of a virus or disease through a social network. We describe these aspects and end with a short discussion on simulation results that inform public policy.
\end{abstract}

Keywords: Computational epidemiology, Discrete dynamical systems, Synthetic populations, Datadriven social network generation, Large-scale stylized network construction, Agent-based simulation, High performance computing

\section{Introduction}

With an increasingly connected world, the potential for large scale virus outbreaks, including epidemics and pandemics, grows. Example outbreaks include severe acute respiratory syndrome (SARS) in 2003, which claimed 800 lives $^{47,126}$; the 2015 Ebola outbreak (8833 killed to date) ${ }^{45}$; and Asian and Hong Kong influenza, each producing death tolls in the millions ${ }^{126}$, which are some of the worst outbreaks in recent history ${ }^{79}$. Over the last 40 years in the U. S., seasonal influenza has caused between 3000 and 49000 deaths annually ${ }^{46}$. Of course, the current COVID-19 pandemic is changing the world health state too rapidly to give "current" statistics.

While deaths and serious illness are immediate impacts of disease outbreaks, there are others. For example, the effects of virus outbreaks on economic costs have been studied ${ }^{15}$, illustrating that interventions can significantly reduce these costs. Secondly, shorter-term conditions can lead to longer-term trends. The so-called poverty trap is the phenomenon wherein poor economic conditions and disease prevalence can lock a society into persistent states of poor health and wealth ${ }^{43}$. A third example is the effect of disease on government instability and upheaval. ${ }^{93}$ provide a regression analysis showing that civil wars may be precipitated or exacerbated by disease outbreaks, because they decrease social health and welfare. Finally, persistent disease threat can also lead to different type of crimes ${ }^{120}$. There are several social issues coming to the fore through the 2019-initiated COVID-19 outbreak. These include closing businesses ${ }^{31}$, educational impacts ${ }^{117}$, disproportionate burden of racial minorities (e.g., in the United States) and by the poor ${ }^{44,63}$, general heightened anxiety levels ${ }^{53,125}$, and domestic violence ${ }^{80,124}$. Hence, there are many reasons to study epidemics and disease outbreaks.

Epidemiologists and the health sciences community use various tools to anticipate outbreaks and help them react to those in progress, as well as perform research to understand disease dynamics and the factors that influence their spread (e.g., $\left.{ }^{16}\right)$. Software simulation tools are used for these purposes and many studies have been conducted (e.g., ${ }^{26,40,49,57,59,72,73,114,133}$ ).
Self Employed, Boston, USA

${ }^{2}$ Lawrence Livermore National Laboratory, Livermore, USA

${ }^{3}$ Texas A\&M UniversityKingsville, Kingsville, USA

${ }^{4}$ University of Virginia, Charlottesville, USA. *hugo3751@gmail.com 
Simulation may use ordinary differential equation (ODE) approaches that, for example, focus on groups (compartments) of people and compute the aggregated numbers of individuals that are in each state (e.g., infected, not infected) as a function of time. These equations essentially describe how many people move from one state to another based on rate equations that involve the current number of people in each compartment. They can also be used to compute characteristics of populations such as the basic reproductive number. See ${ }^{75}$ for a detailed treatment and ${ }^{42,54,57,68,114,115}$ for particular applications and overviews.

Agent-based models $(\mathrm{ABM})^{56,65,67,112}$ represent another class of simulation wherein each human of a population is modeled as a distinct entity or agent that is attributed with traits and behaviors. These agents interact, thereby generating opportunities for contagion transmission among them. Agent interactions on a local scale produce population-level outcomes, such as numbers of cases, regions of high outbreak intensity, subpopulations with greater or lesser numbers of outbreaks, and pathways of virus transmission, among other characteristics. While ODE (aggregated) and ABM (disaggregated) methods each have their strengths, one strength of $A B M$ is the fine-grained opportunities it offers to modify agent traits, behaviors, interactions, and disease parameters and assess their effects on outcomes ${ }^{15}$. Interventions and sensitivity analyses are two example classes of studies that make use of these refinements. Works on use of agentbased modeling for epidemic simulation incl ude $^{3,59,73,76,86,89,100,133}$.

With this background, we turn to the focus of this work in the next section. This paper addresses many disciplines that comprise epidemiological study (in addition to the epidemiological modeling approaches mentioned above). All such disciplines are not covered. Those that are covered are large and wide-ranging. Several references are provided for each discipline; they should be taken as representative, not exhaustive. The topics covered are integrated to give a unified perspective.

We note that while the theme of this paper is computational epidemiology, most of the topics are applicable to other types of human behavior.

\section{An Integrated Modeling Methodology: Scope and Motivation}

Our focus is an ABM environment for computational epidemiology. Agent-based modeling of the spread of viruses or diseases is often given the most attention because it is most closely associated with the ultimate results. However, there are many technical contributions to these final results. Our goals are the construction and use of tools and methods that ultimately produce time-resolved state transitions of each agent in a population, and a quantitative understanding of the factors that produce these results. These tools and their results may then be used by policy analysts and makers ${ }^{41}$. We describe several components of this methodology: (i) theoretical foundations; (ii) population generation procedures and data; (iii) social network construction from these populations; (iv) large-scale stylized network generators; and (v) simulation software that models virus transmission.

There is ample motivation for these individual components, and for their combination and integration. Figure 1 provides an overview of the components and their interactions. We use a formalism called graph dynamical systems (GDS) to study the transmission of diseases and other phenomena. This not only guides simulation software implementations, but also provides a framework to reason about such systems and applications. Examples are provided below. Diseases and other contagions propagate within populations and hence the generation of representative synthetic populations (also called digital twins), down to the individual level, is a key technology. There are many approaches for generating agent-level populations. Social contact networks, and other types of interaction networks, can be produced from these populations. Large scale stylized networks with particular properties, such as scale-free and exponential decay degree distributions, clustering coefficient distributions, assortativity, and community structure, are useful in that dynamics on them can serve as null model results. Moreover, network properties-through different network instances-can be systematically varied in stylized networks as another dimension to sensitivity and parametric studies; this is very difficult to do with synthetic populations. These results can then be compared against those from population-based networks. Disease (contagion) dynamics are computed on these networks using various simulation tools.

The goals of these efforts are to: (i) understand baseline population behavior, (ii) quantify the effects on results of small changes in inputs (sensitivity studies) and of larger changes in inputs (parametric studies), (iii) determine the effects of different interventions, (iv) explain 


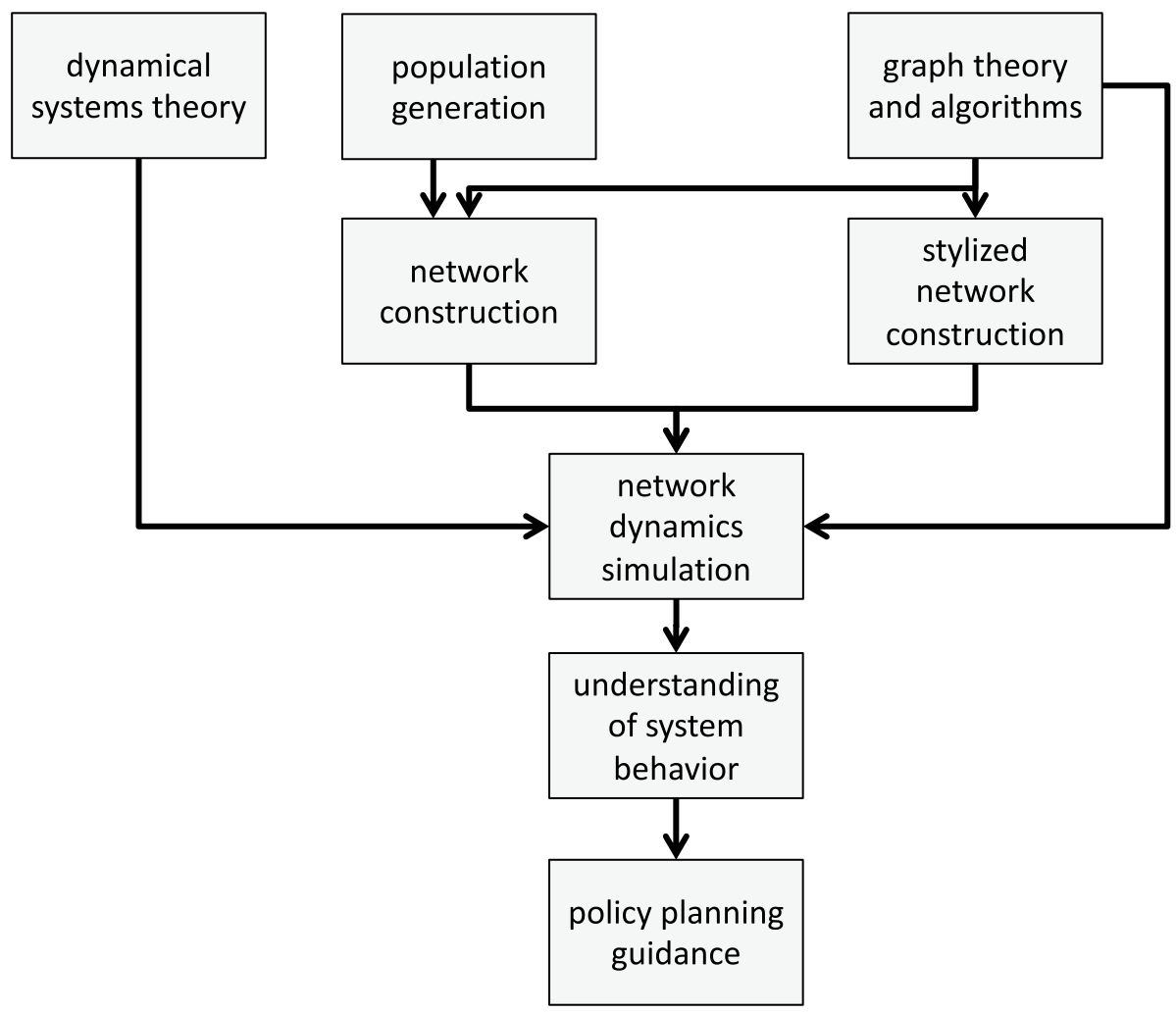

Figure 1: Major components of the agent-based modeling system for computational epidemiology. These boxes are the topics in Sect. 4 below. Feedback loops are not shown. For example, based on the results of system behavior, the population construction methods may be changed, stylized networks with different properties may be generated, or parameters of the dynamics model may be changed.

behaviors and establish causality, and (v) understand results in terms of policy implications.

It is useful to recall that the sizes of networks are routinely on the order of 10s or 100s of millions, or even billions, of agents (nodes) and 100s of millions or billions of edges. Hence, for many of these investigations, parallel processing is required to compute quantities efficiently.

\section{Technical Challenges}

There are many challenges in developing $A B M$ systems. First, we seek a theoretical approach to describe disease dynamics and for this we use graph dynamical systems ${ }^{105}$. Second, to construct populations, data are required from multiple sources. These data are by their nature incomplete, often at different levels of granularity, and may be contradictory. Data fusion under these conditions is challenging. Third, big data challenges exist for generating populations that may involve 10s of millions of agents or more, and 100 s of millions or billions of interactions ${ }^{38}$. In the same way, simulating dynamics on these networks requires parallel computations to drive down execution time and to enable runtime storage of large populations. Hence, efficient simulation is another challenge. A major part of a dynamics evaluation is sensitivity studies: how changes in input parameters affect the results. These require many simulation runs, increasing the need for fast simulation of large populations.

\section{Modeling Environment}

We describe four of the main elements of our modeling environment.

\subsection{Graph Dynamical Systems}

First we provide a theoretical overview of GDS, and then we make the ideas concrete through some examples. Then, we briefly touch on analysis problems and dynamical systems characterizations that are solved using GDS.

\subsubsection{Theoretical Foundations}

We use a discrete dynamical systems formulation known as graph dynamical systems (GDS) $)^{4,97,105}$ 
to model epidemiological processes. A GDS $\mathcal{S}(G(V, E), F, K, W)$ describes the system and its dynamics. Here, $G$ is a (dependency) graph with vertex set $V$ and edge set $E$. We use undirected dependency graphs in this work, but the concepts extend naturally to directed graphs. Each agent $v \in V$ is assigned a state $x_{v} \in K$, where $K$ is the vertex state set. The (system) state $x$ is given by $x=\left(x_{1}, x_{2}, \ldots, x_{n}\right)$ where $n$ is the number of agents in the system; i.e., $n=|V|$. A GDS will have $|K|^{n}$ (system) states. Let $n[v]$ be the sequence of vertex IDs for $v$ itself and for all of its distance- 1 neighbors (i.e., the vertices adjacent to $v$ ), ordered in increasing numerical order. This sequence of vertices is identified from the connectivity of $G$. We denote the states of $v$ and of all of its distance- 1 neighbors as $x[v]$, such that $x[v]=\left(x_{n[v](1)}, x_{n[v](2)}, \ldots, x_{n[v]\left(d_{v}+1\right)}\right), \quad$ where $d(v)$ is the degree of $v$.

A vertex function $f_{v}$ is assigned to each agent $v$ that describes the state transitions for it. The vertex functions for all $n$ agents in the system comprise the sequence $F=\left(f_{v}\right)_{v=1}^{n}$. For each $f_{v}$, the argument is $x[v]$, such that the next state of $v$, denoted $x_{v}^{\prime}$, is given by $x^{\prime}=f_{v}(x[v])$.

Parameter $W$ describes the order in which the vertex functions execute. We will cover two of the most common update schemes: synchronous and sequential.

A synchronous GDS map $\mathbf{F}: K^{n} \rightarrow K^{n}$ is defined by

$$
\mathbf{F}=\left(f_{1}(x[1]), f_{2}(x[2]), \ldots, f_{n}(x[n])\right) .
$$

That is, all vertex functions execute simultaneously. This is also called a parallel GDS map or a synchronous dynamical system. The system state at time $t+1, x(t+1)$, is given by $x(t+1)=\mathbf{F}(x(t))$.

A sequential GDS map $\mathbf{F}_{\pi}$ uses a permutation $\pi=\left(\pi_{1}, \pi_{2}, \ldots, \pi_{n}\right)$ from the set $S_{X}$ of all permutations of the vertices in $V$, where each $\pi_{i} \in V$. We introduce the $X$-local function $F_{v}: K^{n} \longrightarrow K^{n}$, for a vertex $v$, given by

$$
\begin{aligned}
& F_{v}\left(x_{1}, \ldots, x_{n}\right) \\
& \quad=\left(x_{1}, \ldots, x_{v-1}, f_{v}(x[v]), x_{v+1}, \ldots, x_{n}\right) .
\end{aligned}
$$

That is, only the vertex function for $v$ is executed, with the states of all other vertices remaining unchanged. The sequential GDS map $\mathbf{F}_{\pi}: K^{n} \longrightarrow K^{n}$, is then the composition of the $X$-local functions; i.e.,

$$
\mathbf{F}_{\pi}=F_{\pi_{n}} \circ F_{\pi_{n-1}} \circ \ldots \circ F_{\pi_{2}} \circ F_{\pi_{1}} .
$$

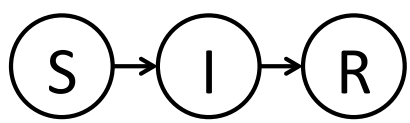

Figure 2: State transition diagrams for the susceptible-infected-recovered (SIR) model. The state set $K=\{S, I, R\}$. Vertex functions quantify the conditions under which a vertex changes state.

This is also referred to as a sequential dynamical system. The system state at time $t+1, x(t+1)$, is given by $x(t+1)=\mathbf{F}_{\pi}(x(t))$.

A forward trajectory is the sequence of (system) states $\left(x(0), x(1), x(2), \ldots, x\left(t_{f}\right)\right)$ through which the GDS evolves from an initial state $x_{0}=x(0)$ to a final state $x\left(t_{f}\right)$, corresponding to a specified end time $t_{f}$. Thus, $x(1)=\mathbf{F}(x(0))$, $x(2)=\mathbf{F}(x(1))=\mathbf{F}^{2}(x(0))$, and so on, until $x\left(t_{f}\right)=\mathbf{F}\left(x\left(t_{f}-1\right)\right)=\mathbf{F}^{t_{f}}(x(0))$ is computed. Consequently, time $t \in\left[0, t_{f}\right]$. A forward trajectory is sometimes referred to as a diffusion instance. If, for a deterministic system map $\mathbf{F}$ -one in which there is precisely one value of $x(t+1)=\mathbf{F}(x(t))$ for each $x(t)$-we have that $x(t+1)=x(t)$, for some time $t$, then the GDS has reached a fixed point. That is, when a fixed point is reached at time $t^{*}, x(t)=x\left(t^{*}\right)$ for all $t \geq t^{*}$; the system state does not change. Further, if there exists a smallest integer $q$ such that $x(t+q)=x(t)$, and no $q^{*}<q$ exists such that $x\left(t+q^{*}\right)=x(t)$ for some $t$, then the long-term dynamics is a repeating sequence or cycle of states $(x(t), x(t+1), \ldots, x(t+q-1))$ called a limit cycle with cycle length $q$. When $q=1$, the limit cycle is a fixed point.

A GDS may be deterministic or stochastic. We say that $x(t+1)$ is the successor of $x(t)$ and that $x(t)$ is the predecessor of $x(t+1)$. In a deterministic system, a state $x$ may have many (or zero) predecessors, but only one successor (which may be itself). In a stochastic system, a state may have any number of predecessors and will have at least one successor, up to $|K|^{n}$ predecessors and successors. All of these states may or may not be visited in one forward trajectory of deterministic and stochastic GDSs.

Typically, for computations on large populations, the synchronous update mechanism is used to take advantage of parallel processing capabilities of simulation software that incorporates parallel execution of vertex functions. 


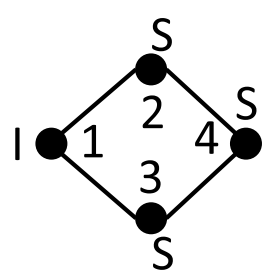

$\mathrm{t}=0$

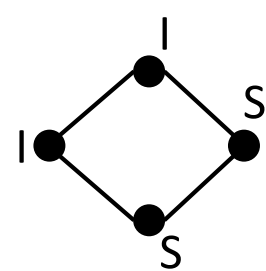

$\mathrm{t}=1$

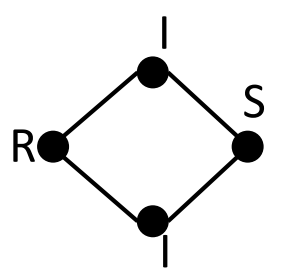

$\mathrm{t}=2$

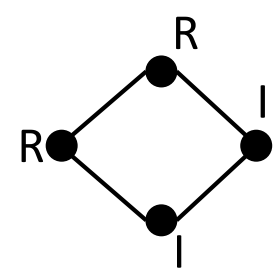

$t=3$

$(I, S, S, S)$

$(I, I, S, S)$

$(\mathrm{R}, \mathrm{I}, \mathrm{I}, \mathrm{S})$

$(R, R, I, I)$

Figure 3: Illustrative example of a 4-time step forward trajectory for a synchronous GDS where each vertex function is an SIR model. The dependency graph has 4 vertices and 4 edges. The infectious duration for each vertex (agent) is $t_{d l}=2$, and the probability of infection is $p$ for each vertex. The vertices (agents) are labeled in the graph at the left, corresponding to the initial state $x(0)$. The state corresponding to each time, displayed below the time, is given as $x(t)=\left(x_{1}, x_{2}, x_{3}, x_{4}\right)$. This particular sequence of states is dependent on the random numbers $r_{i}, 1 \leq i \leq 5$, and their relation to $p$.

\subsubsection{Example: SIR Model With Explicit Dependency Graph}

The state transition diagram for the susceptibleinfectious-recovered (SIR) model is provided in Fig. 2. Vertex functions $\left(f_{v}\right)_{v=1}^{n}$ quantify the conditions under which the two state transitions $S \longrightarrow I$ and $I \longrightarrow R$ take place. For the sake of simplicity in this example, we let $p \in[0,1]$ be a probability of infection that holds for all agents (in many cases, $p$ may be a function of simulation variables, such as the duration of contact between an infectious agent and a susceptible one). We let $t_{d I}$ be the duration (i.e., number of time steps) that a vertex spends in the infectious (I) state; again, we take this as uniform across all agents, but in practice can vary among agents. Let $t_{i v}$ be the time at which vertex $v$ transitions to state $I$. A description of the vertex function follows, in which a vertex $v$ transitions from $x_{v}$ to $x_{v}^{\prime}$ :

\section{Vertex function $f_{v}$ for node $v$}

1. If $x_{v}=R$, then $x_{v}^{\prime}=R$, irrespective of the states of $v$ 's neighbors.

2. If $x_{v}=I$ and $t_{i v}$ was the time at which this agent $v$ was infected, then if the current time $t=t_{i v}+t_{d I}$, then $x_{v}^{\prime}=R$. Otherwise, $v$ does not change state; i.e., $x_{v}^{\prime}=x_{\nu}$.

3 . If $x_{v}=S$, then for each neighbor $u$ of $v$ that is in state $I, v$ transitions to state $I$ with probability $p$. If $v$ does not change state, then $x_{v}^{\prime}=x_{v}$.

In particular for Condition 3, only one neighbor of $v$, in state $I$, is needed to cause $v$ to change to state $I$. This is an example of a stochastic GDS, because, owing to the probability $p$, there is not a unique $x(t+1)$ given $x(t)$.

Figure 3 provides a simple example that illustrates the dynamics of an SIR-based GDS. Each vertex is an agent, so the graph is a person-to-person contact or interaction graph. Each vertex function is the SIR function given immediately above. Let $t_{d I}=2$. The states at four times are shown: $x(0), x(1), x(2)$, and $x(3)$. Initially, only vertex 1 is infected; all other vertices are in state $S$. At time $t=1$, a random number $r_{1}<p$ is generated for vertex 2 , based on its edge with vertex 1 , resulting in 2 transitioning to state $I$. For vertex 3 and its edge to vertex $1, r_{2}>p$ and hence 3 does not change state. Vertex 4 has no neighbor in state $I$ and thus remains in state $S$. At time $t=2$, vertex 3 changes to state $I$ because, based on vertex 3's edge with vertex 1 , the generated random number $r_{3}<p$. Vertex 4 does not change state. Vertex 1 transitions to state $R$ at the end of time $t=2$. Other state changes occur similarly. In this example, the dependency graph is explicit. In the next example, it is implicit.

\subsubsection{Example: SIR Model With Implicit Dependency Graph}

In the previous example, we used an explicit agent-to-agent social network for the dependency graph. In this example, we use a different type of graph, shown in Fig. 4. It is a bipartite peoplelocations graph (PLG), where one bipartition is the set of vertices representing people and the other bipartition is the set of vertices representing locations ${ }^{59}$. The edge labels are the times of the 
people

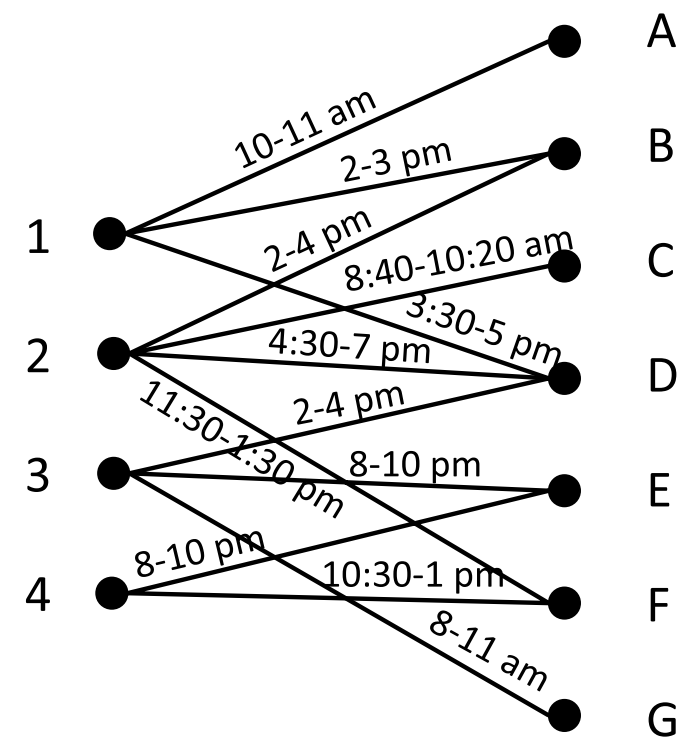

Figure 4: Illustrative example of a person-location bipartite graph, where people (1 through 4) are the elements of the left partite set and locations (A through $\mathrm{G}$ ) are the elements of the righ partite set. The edges are labeled with time When two people are co-located, they form a person-person edge in a social network such as that in Fig. 3. Since these interactions give rise to the same person-person edges as in Fig. 3, and the same SIR model vertex functions are used, this network produces the same forward trajectory as that in in Fig.

day at which the person is located at the specified location.

A person-to-person contact graph is produced in the following way: an edge between two people is generated if they appear at the same location, at the same time. We say that they are co-located. For example, consider persons 1 and 2 in Fig. 4. They are co-located at location $\mathrm{B}$ between 2 and $3 \mathrm{pm}$, and at location D from 4:30 to $5 \mathrm{pm}$. This results in one edge in the person-person graph in Fig. 3. Persons 1 and 3 also form an edge from the interaction at location $\mathrm{D}$, because they overlap from 3:30 to $4 \mathrm{pm}$. Proceeding with this construction, we recover the dependency graph of Fig. 3 .

In this example, the SIR model is the same as that in Sect. 4.1.2, and since we use the same synchronous update scheme and assume that the random numbers are the same as those in Sect. 4.1.2, we produce the same forward trajectory as above in Fig. 3.
There are variants of this approach. For example, the probability $p$ of interaction can be a function of the contact duration between two people. In both examples, edges can be time-varying. For the first example, the edges can have labels denoting their interaction times, in a fashion similar to that in Fig. 4. In the second example, the activity pattern of an agent may change. An activity pattern is the set of locations that a person visits, along with the times of the visits.

\subsubsection{Analysis Problems and System Characterizations}

Besides providing a framework for constructing simulation software, GDS also provides a framework for investigation of analysis problems and dynamical systems characterization. Analysis problems of interest here are those regarding system dynamics of the types described in this section. The following are some examples:

1. Reachability problems. Given a state $x$, can the state $x^{\prime}$ be reached in one time step? More generally: given a state $x$, can state $x^{\prime}$ be reached in at most $t$ time steps?

2. Predecessor existence problems. Given a state $x$, is there a state $x^{\prime}$ such that the system transitions from $x^{\prime}$ to $x$ in one time step? More generally: given a state $x$, is there a state $x^{\prime}$ such that a GDS transitions from state $x^{\prime}$ to $x$ in at most $t$ time steps?

3. Fixed point existence problems. Given a GDS, does it have fixed points? A counting version is: How many fixed points does a system have?

These types of problems and their answers are important for practical reasons. For example, if a given state $x$ of a system has been observed, one may want to know whether a particularly harmful state $x^{\prime}$ can arise; this is an example of a reachability problem. We refer the reader to numerous works on these and other related analysis problems. See ${ }^{17-21,21,22,24,25,28,82,116,122}$.

The GDS framework also provides a foundation for characterizing dynamical systems. Useful texts include ${ }^{69,105}$. An example of a system characterization is the following: progressive Boolean threshold systems, heavily used in the social sciences ${ }^{48,71,119}$ and that are similar to models popularized in $^{78}$, are shown to generate only fixed points as limit cycles ${ }^{85}$. Works with other characterizations include ${ }^{2,69,83,84,105,121,127}$. These types of results, along with those from analysis 
problems, are useful not only for understanding system dynamics, but also for modeling and simulation verification and validation.

\subsection{Synthetic Population Generation}

A synthetic population is a (data) representation of a group of individuals. The notion of group varies widely, from the members of a single family to all of the people of a nation. The wide range in sizes of such populations is a signal of their increasing development and use. Synthetic populations are also called "digital twins." The individuals in a synthetic population are often endowed with (demographic) traits, such as age, gender, home location and housing. They are often given activity patterns where individuals go to particular locations and particular times of days. There is often additional data associated with synthetic individuals; particular data (assigned to synthetic individuals) depends on the requirements and use of the population. Figure 5 is a conceptual view of a synthetic population and movements of individuals as they perform daily activities; the two types of networks described above; and an attributed individual.

Typically, these populations are not one-toone with actual populations. In other words, consider a real person who lives in a real city in the United States, on a particular street, with a family. That person is not (typically) represented in a synthetic population. Rather, distributions of characteristics of a synthetic population match those of the actual population. For example, age and gender distributions of people within a U.S. state, and distributions of household sizes are matched within a synthetic population. Because of the stochastic nature of the synthetic population construction process, one synthetic population is typically one instance or realization of a family of instances. Work has been done to assess the variability of synthetic population instances, e.g., ${ }^{61}$.

\subsubsection{Synthetic Populations and Their Building Blocks}

The synthetic population generation process is comprised of the following steps ${ }^{13,39}$.

Constructing synthetic individuals and households. Individuals and households (collections of individuals) are created. Individuals are endowed with characteristics such as age, gender, marital status. A representation of each household is created from census data by collecting individuals and assigning attributes such as household income and size.

Determining activities of individuals. Each synthetic person in a household is assigned a set of activities to perform during a day, along with the times when the activities begin and end, as given by activity or time-use survey data.

Determining locations for activities. An appropriate real location is chosen for each activity of every synthetic person based on a gravity model (i.e., locations closer to home locations are more likely to be selected, but longer distance locations are also selected, just with lesser probability) and data sources such as land use patterns, tax data, or commercial location data. Locations often have sublocations (e.g., sublocations may be rooms within a building), so that a location may be a (location, sublocation) pair.

Generating social contact networks. Two different representations of human interaction networks are provided. See the discussions for Figs. 3 and 4 .

Below we describe each of the four steps above in more detail, for populations generated for regions of the U.S.

\subsubsection{Constructing Synthetic Individuals and Households}

A baseline population is constituted from two data sources: American Community Survey (ACS) and Public Use Microdata Sample (PUMS). The ACS data are used to create an individualized set of agents with assigned characteristics; see ${ }^{37}$ for details. The PUMS data are used to construct individuals that have, in distribution, the same traits as those of members of an actual population.

The ACS provides data for public use that are resolved to the block group level, which is a geographical region containing between 600 and 3000 people. For each block group, tables of distributions of many demographic characteristics—-such as age, gender, and household sizeare provided. These are marginal distributions. To create a synthetic population for the block group, a joint distribution is constructed from the given marginal distributions. This distribution is sampled the required number of times (one for each member of the target population).

The ACS also provides a 5 percent representative sample for each region, known as a PUMS, which is generated from a larger region called a Public Use Microdata Area (PUMA); the latter contains at least 100,000 people. A PUMS record 

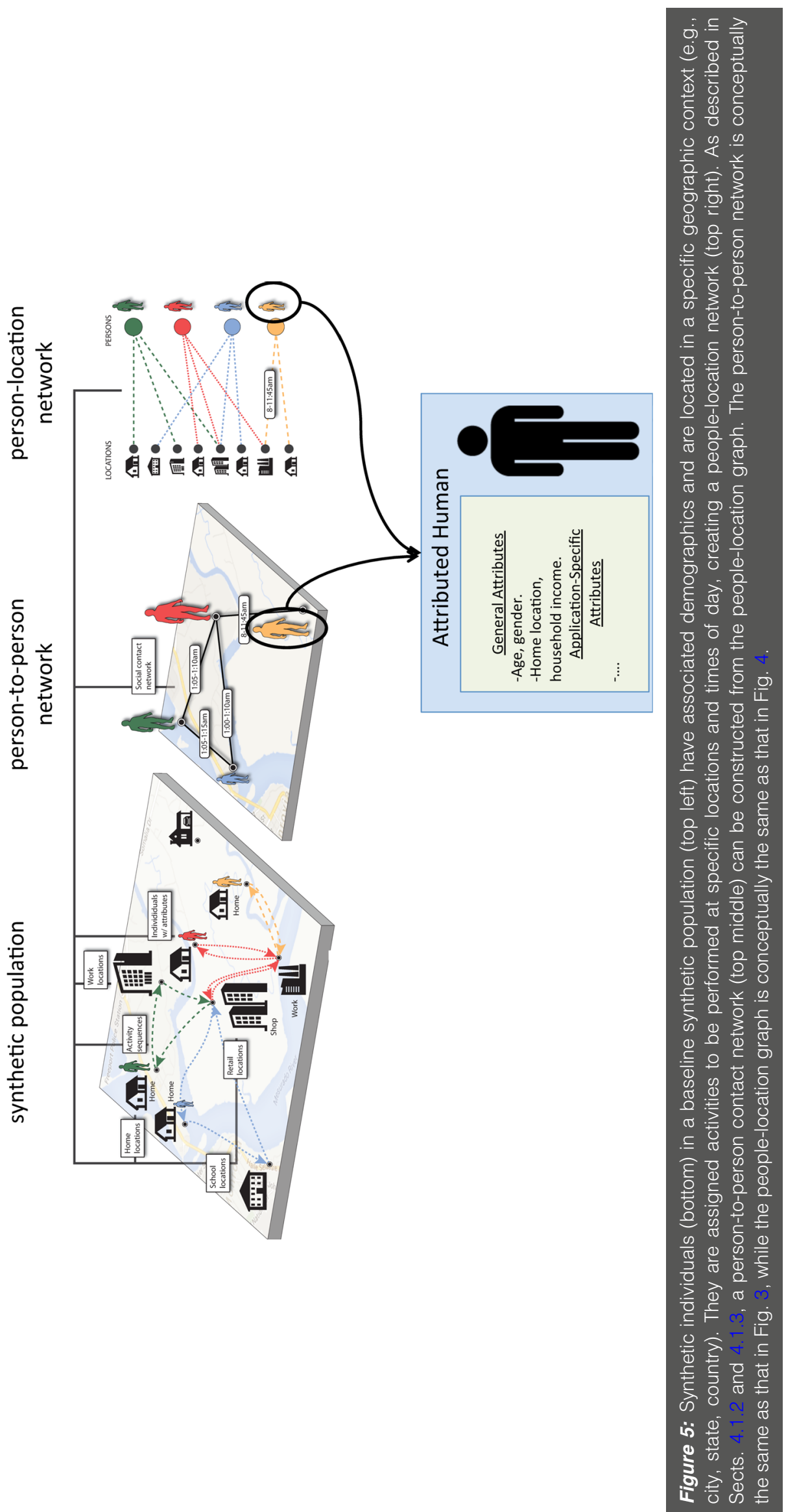
Table 1: Characteristics of selected U.S. city

populations, in millions ${ }^{13}$.

\begin{tabular}{llll}
\hline City & $\begin{array}{l}\text { Number } \\
\text { of agents }\end{array}$ & $\begin{array}{l}\text { Number } \\
\text { of locations }\end{array}$ & $\begin{array}{l}\text { Number } \\
\text { of edges }\end{array}$ \\
\hline Los Angeles & $16.2 \mathrm{M}$ & $3.2 \mathrm{M}$ & $917 \mathrm{M}$ \\
New York City & $17.9 \mathrm{M}$ & $4.3 \mathrm{M}$ & $961 \mathrm{M}$ \\
Seattle & $3.2 \mathrm{M}$ & $0.78 \mathrm{M}$ & $177 \mathrm{M}$ \\
\hline
\end{tabular}

is essentially a complete census record. The PUMS information is incorporated into the inference of the joint distribution from the marginal distributions using a statistical procedure called iterative proportional fitting (IPF) ${ }^{51,55}$. IPF is an approach for combining the information from the marginal distributions and the sample data. It has been shown to preserve important properties and correlations of the data; $\operatorname{see}^{77,94}$. The joint distribution for each block group is sampled to select households (with individuals) from the PUMS data, and these households are added to the synthetic population for that block group.

\subsubsection{Determining Activities of Individuals}

Data from the National Household Travel Survey (NHTS) are used to assign a daily activity sequence to each individual in a synthetic population. The NHTS contains detailed information on individual's movements and activities over the course of a normative day ${ }^{118}$.

The activity patterns for different members of a household are typically dependent on each other. For instance, if there is a child under twelve years of age in the household, then an adult will likely be present in the home with the child whenever the child is at home.

NHTS surveys households, not individuals. Consequently, activity sequences are assigned by household, for each household, thereby preserving within-household activity correlations. This method is known as the Fitted-Values Method or $\mathrm{FVM}^{95}$. Essentially, a survey household is selected that is similar to the synthetic household using the asymmetric Hausdorff distance; a person within the survey household is selected that is most similar to each synthetic person; and that survey person's activities are assigned to the synthetic individual, for each household member.

\subsubsection{Determining Locations for Activities}

Since each activity must be located, there are procedures for assigning (i) home locations and (ii) locations for other activities.

Home locations are assigned with the following procedure. U. S. Census data provide geographic boundary data (shapefiles) for each block group. The Census and ACS provide housing unit distributions (number of buildings with that contain different numbers of housing units), again for each block groups. HERE contains road networks, including geographic information. The portions of road networks that lay within the boundary of a block group are determined. Residential locations within a block group are assigned along these roadways with, e.g., single family homes more likely to be assigned to smaller (less traveled) streets. Households are assigned to these home locations.

Locations for other activities for individuals are assigned using a gravity model (see, e.g. ${ }^{81,123}$ for works on gravity models). The idea is that the probabilities of assigning particular locations for the next activity in a synthetic individual's time-order list of activities are proportional to the capacities of buildings and inversely proportional to the distances from the current location to the candidate next locations. The base location is a person's home location. From the determined current location of the most recent activity, it is more likely that a closer location of greater human capacity is chosen for the next activity's location. For schools, National Center for Education Statistics (NCES) $)^{109,110}$ information is used; for business and other activity locations, $\mathrm{D} \& \mathrm{~B}$ data are used. See $e^{35,59,70}$ for additional information on location assignments. The above location assignments are constrained by capacities that are assigned to each building, or rooms (i.e., sublocations) within buildings. Consequently, a location may be a (location, sublocation) pair.

\subsubsection{Generating Social Contact Networks}

Each person has been assigned a (location, sublocation) pair for each activity during the day. Each activity has a start and end time. Thus, all information for the network representation in Sect. 4.1.3 is known, and a person-location graph analogous to that in Fig. 3 can be generated for a synthetic population. To generate a personperson contact social network, a graph edge is formed between two synthetic individuals (i.e., two nodes in the network) if they are located at 
the same (location, sublocation) with overlapping visit times during the day. Sect. 4.1.2 describes such as person-person network. Aspects of contact network evaluation can be found at ${ }^{59,60,128,129}$. Illustrative examples of populations and their contact networks are provided in Table 1. It is evident that populations with billions of edges (e.g., for states and countries) are readily attainable.

\subsubsection{Other Population Generation Approaches} In concluding this section, we note that there are several other approaches for generating and evaluating synthetic populations, and there are many applications. See for examples $^{10,30,64,74,91,96,102,104,106-108,130,131,135}$. Reviews can be found $\mathrm{at}^{29,50,113}$.

\subsection{Stylized Network Construction}

Constructing explicit contact networks on the involved individuals allows us to study disease dynamics in more detail than possible with some other methods (e.g., compartmentalized models). A contact network is a graph $G(V, E)$, where $V$ is the set of persons (each person is a vertex) and $E$ is the set of contacts or edges; each edge $(u, v) \in E$ indicates the existence of the contact between two persons $u$ and $v$. Use of a social contact network, in which a link represents physical contact between two people, can provide greater understanding of the disease dynamics. However, such studies require explicit networks, in which contacts (edges) exist explicitly. Although the data for these networks is difficult to get because of privacy and security concerns, conceptually these networks are well-defined. A study of epidemics (e.g., influenza, which spreads by physical contact) requires social contact networks, in which an edge represents an actual physical contact between two people at some location during the day. Procedures for generating these networks were discussed in Sect. 4.2.

In this section, we discuss several procedures for generating large stylized networks. As mentioned earlier, these networks are useful for several reasons. First, because (selected) properties of these networks can be controlled, the effects of network structure on disease dynamics (see Sect. 4.4) can be quantified. Second, even within a class of graphs, variations in parameter settings for their construction can also be evaluated to determine their effects on disease dynamics. Third, results of virus spreading on these networks can be used as null model results that can be compared with those generated with the networks of the preceding section.

Often, contact networks are approximated by various random network models such as the Erdös-Rényi model $^{58}$, preferential attachment model $^{9,11}$, Chung-Lu model ${ }^{103}$, etc. The ErdösRényi model is the most widely-used and wellstudied model due to its simplicity. Its simplicity has allowed us to perform rigorous theoretical analysis on this model over the last several decades. However, the Erdös-Rényi model generates random networks that have binomial degree distributions, which are not common in the real world. The preferential attachment model produces networks with power-law degree distributions. Many real-world networks follow power-law degree distributions, but many do not. Chung-Lu is a more general model that can produce a network from any given degree distribution. However, a degree distribution must be input to the Chung-Lu model whereas ErdösRényi and preferential models are controlled using just a few scalar parameters. In this section, we discuss some details of these models.

The study of complex systems has significantly increased the interest in various random graph models $7,33,66$. As some of the complex networks grow, it has become necessary to correspondingly generate massive random networks efficiently. As discussed $i^{92}$, the structure of larger networks is fundamentally different from small networks, even if both are generated using the same model, and many patterns emerge only in massive graphs. Demand for large random networks necessitates the use of efficient algorithms, in terms of both running time and memory consumption, for their generation. Therefore, in addition to the description of these models, we also discuss some efficient sequential and parallel algorithms for generating random networks using the models.

Although various random graph models are being used and studied over the last several decades, even efficient sequential algorithms for generating such graphs were nonexistent until recently. Batagelj and Brandes ${ }^{33}$ justifiably said "To our surprise we have found that the algorithms used for these generators in software such as BRITE, GT-ITM, JUNG, or LEDA are rather inefficient. ...superlinear algorithms are sometimes tolerable in the analysis of networks with tens of thousands of nodes, but they are clearly unacceptable for generating large numbers of such graphs." As a step towards meeting this goal, efficient sequential algorithms have recently been developed to generate certain classes of random 

00
$(0,1)(0,2)(0,3)$
$\begin{array}{ccc}0 & \bigcirc & \bigcirc \\ (1,2) & (1,3) & (1,4)\end{array}$
- 0
$(2,3)(2,4)(2,5)$

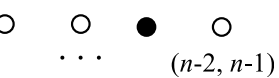

Figure 6: A sequence of all possible potential edges. Each circle represents a potential edge. The white circles are the skipped edges, and the solid black circles are the selected edges in an Erdös-Rényi graph.

graphs: Erdös-Rényi ${ }^{33}$, small world ${ }^{33}$, Preferential Attachment ${ }^{33,111}$, and Chung-Lu ${ }^{103}$. Although these efficient sequential algorithms are able to generate networks with millions of nodes quickly, generating networks with billions of nodes can take substantially longer. Further, a large memory requirement often makes generation of such large networks using these sequential algorithms infeasible. Thus, parallel algorithms that scale to large numbers of processors and provide good speed up become a necessity.

The design of parallel distributed memory algorithms poses two main challenges in the context of generating random graphs. First, the dependencies among the edges, especially in the preferential-attachment model, impede independent operations of the processors. Second, different processors can create duplicate edges, which must be avoided. Dealing with both of these problems requires complex synchronization and communication among the processors, and thus gaining satisfactory speedup by parallelization becomes a challenging problem. Even for the Erdös-Rényi model where the existence of edges are independent of each other, parallelization of a non-naive efficient algorithm, such as the algorithm by Batagelj and Brandes ${ }^{33}$, is a nontrivial problem. A parallelization of Batagelj and Brandes's algorithm was recently proposed in ${ }^{111}$.

\subsubsection{Erdos-Renyi Networks}

The Erdös-Rényi model ${ }^{58}$ is well-studied and one of the first random graph models. The model is as follow. We are given two parameters: an integer $n$ and a real number $p$ in $[0,1]$. The model generates a random graph with $n=|V|$ vertices such that for every pair of vertices $u, v \in V$, edge $(u, v)$ is included in the graph independently at random with probability $p$. Since there are $\left(\begin{array}{l}n \\ 2\end{array}\right)$ possible pairs of nodes, the expected number of edges is $\frac{1}{2} n(n-1) p$ and the expected degree of each vertex is $(n-1) p$. It is easy to see that the degree distribution is binomial.

A naive algorithm is: for each pair of vertices $u$ and $v$, pick edge $(u, v)$ independently with probability $p$ by tossing a biased coin. Since there are $\Theta\left(n^{2}\right)$ pairs of vertices, this algorithm takes $\Theta\left(n^{2}\right)$ time. An efficient algorithm is given in $^{33}$ that takes $O(m)$ time, where $m$ is the number of edges in the generated graph. The runtime is improved by avoiding tossing coins for the edges that are not selected. Consider a sequence of all possible edges, that is, all possible pairs of vertices as shown in Fig. 6. If we select each edge with probability $p$ independently, a streak of edges are skipped between two selected edges (solid back circles in the figure). Instead of discarding those edges one by one, their algorithm determines the number of edges to be skipped by generating a single random number using the following geometric distribution. Let $\delta$ be a random variable denoting the number of edge to be skipped. Then $\delta$ edges are skipped and the following edge is selected to be added to the graph. This process is repeated until there is no remaining potential edges. The number edges to be skipped is called the skip length, which is computed as follows. We have

$$
\operatorname{Pr}\{\delta=k\}=(1-p)^{k} p
$$

i.e., $\delta$ is a geometric random variable. A geometric random number can be generated as follow.

$$
\begin{aligned}
& -\quad r \leftarrow \text { a uniform random number in }[0,1) \\
& -\quad \delta \leftarrow\left\lfloor\frac{\log (1-r)}{\log (1-p)}\right\rfloor
\end{aligned}
$$

For additional details see ${ }^{33}$. Since each edge in the generated graph requires one random number and constant time, the algorithm takes $O(m)$ time, which is optimal.

A parallelization of the above sequential algorithm is given $i^{111}$. In the sequential algorithm the edges are selected one after another as the algorithm walks through the sequence of the potential edges (as shown in Fig. 6) using the skip lengths. Notice that determining a selected edge is dependent on the previous selected edges. Thus the process seems to be sequential in nature and pose a difficulty in parallelization. To deal with this difficulty, instead of generating an edge instantly after computing a skip length, all skip lengths are computed and stored by the processors, and then edges are created from these skip lengths. Another difficulty is that the algorithm does not know how many edges, and consequently how many skip lengths, need to generated 
in advance. The algorithm begins with an estimated number of skip lengths $B$. the expected number of edges $\frac{1}{2} n(n-1) p$ can serve as an estimation for $B$. Each of the $P$ processors generates $B / P$ skip lengths and stores them in an array $S$. Then a parallel prefix sum operation on the array $S$ is performed by the processors to generate the actual edges. Let $T$ be the sum of the skip lengths. If $T<\frac{1}{2} n(n-1)$ (i.e., $\mathrm{B}$ is an under estimation), generate some additional skip lengths. If there are some extra skip lengths, they are discard. See ${ }^{111}$ for details.

\subsubsection{Preferential Attachment Networks}

The preferential attachment model generates random evolving scale-free networks using a preferential attachment mechanism: a new node is added to the network and connected to some existing nodes that are chosen preferentially based on some properties of the nodes. In the most common applications, preference is given to nodes with larger degrees: a node is chosen with probability proportional to its degree. Below we discuss this degree-based preferential attachment (PA) model. A random network generated with a PA model has a power-law degree distribution ${ }^{11}$. In a power-law degree distribution, the probability that a node has degree $d$ is given by $\operatorname{Pr}\{d\} \sim d^{-\gamma}$, where $\gamma$ is a positive constant.

Let $n$ be the number of nodes in the network we want to generate. In this model, nodes are added one by one. In phase $t, 0 \leq t<n$, a new node $t$ is added to the network and connected to $x$ randomly chosen existing nodes. In this discussion, we use $x=1$. The methods described below can easily be generalized for any $x \geq 1\left(\right.$ see $\left.^{6}\right)$. Let $F_{t}$ be the node selected in phase $t$, i.e., edge $\left(t, F_{t}\right)$ is added to the network. Let $P_{t}(i)$ be the probability that node $t$ is connected to node $i<t$; that is, $P_{t}(i)=\operatorname{Pr}\left\{F_{t}=i\right\}=\frac{d_{i}}{\sum_{j} d_{j}}$, where $d_{j}$ represents

the degree of node $j$. A naive implementation of this method can be inefficient. Batagelj and Brandes $^{33}$ give an efficient algorithm with running time $O(m)$. This algorithm maintains a list of nodes such that each node $i$ appears in this list exactly $d_{i}$ times. The list can easily be updated dynamically by simply appending $u$ and $v$ to the list whenever a new edge $(u, v)$ is added to the network. Now to find $F_{t}$, a node is chosen from the list uniformly at random. Since each node $i$ occurs exactly $d_{i}$ times in the list, we have $\operatorname{Pr}\left\{F_{t}=i\right\}=\frac{d_{i}}{\sum_{j} d_{j}}$.
Another algorithm, called copy model, proposed in ${ }^{87}$ also leads to preferential attachment and power law degree distribution. The algorithm works as follows. In each phase $t$,

Step 1: first a random node $k \in[1, t-1]$ is chosen with uniform probability.

Step 2: then $F_{t}$ is determined as follows:

$$
\begin{aligned}
F_{t} & =k \text { with prob. } p \\
& =F_{k} \text { with prob. }(1-p)
\end{aligned}
$$

In the copy model when $p=\frac{1}{2}$, we have $\operatorname{Pr}\left\{F_{t}=i\right\}=\frac{d_{i}}{\sum_{j} d_{j}}$,8. Thus, the copy model is

more general. Further, it is easy to see the running time of the copy model is $O(m)$, and it leads to more efficient parallel algorithms.

A parallel algorithm based on the copy model is given $\mathrm{in}^{6,8}$. The dependencies among the edges pose a major challenge in parallelizing preferential attachment algorithms. Apparently any algorithm for preferential attachment seems to be highly sequential in nature: phase $t$ cannot be executed until all previous phases are completed. However, a careful observation reveals that $F_{t}$ can be partially, or sometime completely, determined even before completing the previous phases. Notice that Step 1 above in the copy model can be executed for all node $t$ simultaneously and independently. In Step 2, if $F_{t}=k$, we are done with the computation of $F_{t}$. If $F_{t}=F_{k}$, we may need to wait and coordinate with other processors as described below. Assuming there are $P$ processors, the set of nodes $V$ is divided into $P$ disjoint subsets $V_{1}, V_{2}, \ldots, V_{P}$; that is, $V_{i} \subset V$, such that for any $i$ and $j, V_{i} \cap V_{j}=\emptyset$ and $\bigcup_{i} V_{i}=V$. Processor $P_{i}$ is responsible for computing and storing $F_{t}$ for all $t \in V_{i}$. The load balancing and performance of the algorithm crucially depend on how $V$ is partitioned. See ${ }^{6}$ for a detailed study on load balancing and partitioning of $V$. Let $t \in V_{i}$. Now, if $F_{t}$ is chosen to be $F_{k}$, to determine $F_{t}$, we need to wait until $F_{k}$ is known. If $k \in V_{j}$ with $i \neq j$, processor $i$ sends a message to processor $j$ to find $F_{k}$. If $F_{k}$ is unknown, $P_{j}$ keeps this message in a queue until $F_{k}$ is known. Once $F_{k}$ is known, $P_{j}$ sends back a message with $F_{k}$ to $P_{i}$.

Now notice that while processor $P_{i}$ waits for processor $P_{j}$ until $F_{k}$ is known, it is possible that to determine $F_{k}$, processor $P_{j}$ is waiting for some other processor and so on. These events may lead to a waiting chain or dependency chain (see Fig. 7). If the lengths of the dependency chains are large, it can cause some processors wait for a long time, leading to poor performance of the 


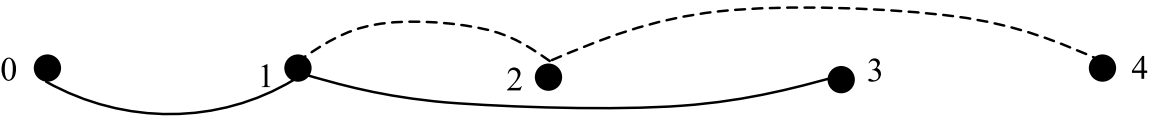

Figure 7: A preferential attachment network with 5 nodes generated using the copy model. Solid lines show final decided edges, and dashed lines denote waiting of processors for node attachment to be resolved - the undecided edges. For node $t=3, k$ is chosen to be $2, F_{3}$ is chosen to be $k=2$, and thus edge $(3,1)$ is decided immediately. Similarly, edge $(1,0)$ is also decided immediately. For node $t=4, k$ is 2 and $F_{4}$ is set to be $F_{2}$. That is, $F_{4}$ is dependent on $F_{2}$. Similarly, $F_{2}$ is dependent on $F_{1}$. Finally, we have $F_{4}=F_{2}=F_{1}=0$.

parallel algorithm. Fortunately, the length of a dependency chain is small, and the performance of the algorithm is hardly affected by such waiting steps. $\mathrm{In}^{6,8}$, it is shown that the maximum length of a dependency chain is at most $O(\log n)$ with high probability. Moreover, while $O(\log n)$ is the maximum length, most of the chains have much smaller length. It is easy to see that for a constant $p$, the average length of a dependency chain is also constant, which is at most $\frac{1}{p}$. For an

arbitrary $p$, the average length is still bounded by $\log n$. Thus, while for some nodes a processor may need to wait for $O(\log n)$ steps, the processor hardly remains idle as it has other nodes on which it can work.

\subsubsection{Chung-Lu Networks}

The Chung-Lu model ${ }^{52}$ generates a random network from a given sequence of expected degrees. We are given a sequence of weights (representing expected degrees of the nodes), the model generates a random network such that the expected degree of a node is equal to the corresponding weight in the given sequence. Let the given sequence of the weights be $\left(W_{0}, W_{1}, \ldots W_{n-1}\right)$, where $W_{v}$ represents the expected degree of node $v$ for all $v \in V=\{0,1, \ldots, n-1\}$, the set of nodes. Assuming $W_{v}^{2}<\sum_{k \in V} W_{k}$ for all $v \in V$, the model works as follows. For every pair of nodes $u, v \in V$, edge $(u, v)$ is added to the network with probability

$$
p_{u, v}=\frac{W_{u} W_{v}}{S}, \text { where } S=\sum_{k \in V} W_{k} .
$$

Now we have the expected degree for each $v$,

$$
\begin{aligned}
E\left[d_{v}\right] & =\sum_{u} p_{u, v}=\sum_{u \in V} \frac{W_{u} W_{v}}{S} \\
& =\frac{W_{v}}{S} \sum_{u \in V} W_{u}=W_{v} .
\end{aligned}
$$

Notice that above model can produce self loops. However, the self loops can easily be avoided by a simple modification of the model. One way to avoid the self loops is to simply discard any self loops created ${ }^{103}$. In such a case,

$$
E\left[d_{v}\right]=\sum_{u \in V, u \neq v} \frac{W_{u} W_{v}}{S}=W_{v}-\frac{W_{v}^{2}}{S} .
$$

For large graphs, where the number of nodes $n$ is very large, the expected degree $E\left[d_{\nu}\right]$ converges to $W_{v}$ for each node $v$. It is also possible to adjust the probability $p_{u, v}$ such that even after discarding the self loops, $E\left[d_{\nu}\right]$ is exactly equal to $W_{v}$.

A naive algorithm for the Chung-Lu model is for each pair of nodes $u, v \in V$, create edge $(u, v)$ with probability $p_{u, v}=\frac{W_{u} W_{v}}{S}$ independently (independent of the other edges). Like the Erdös-Rényi model, this naive algorithm requires $O\left(n^{2}\right)$ time to generate a network with $n$ nodes since there are $\frac{1}{2} n(n-1)$ possible pairs of nodes. The difference between Erdös-Rényi model and Chung-Lu model is that in the Erdös-Rényi model all edges are created with same probability whereas in the Chung-Lu model different edges have different probabilities. An efficient $O(n+m)$ time algorithm is given $\mathrm{in}^{103}$. This algorithm is based on a technique similar to the edge skipping technique used in ${ }^{33}$ for the Erdös-Rényi model. Let the sequence of weights be sorted in non-increasing order. First consider the following algorithm. For each node $u$, pick each edge from the sequence of edges $(u, u+1),(u, u+2),(u, u+3), \ldots$, in this order, with probability $p=p_{u, u+1}=\frac{W_{u} W_{u+1}}{S}$ until an edge $(u, v)$ is picked. Let $q=p_{u, v}=\frac{W_{u} W_{v}}{S}$. Now include edge $(u, v)$ in the generated network $G$ 
with probability $\frac{q}{p}$. Then repeat the above pro-

cess again beginning with edge $(u, v+1)$ and probability $p_{u, v}$. Notice that for any $u, v \in V$, edge $(u, v)$ is included in $G$ with probability $p \cdot \frac{q}{p}=q=p_{u, v}$. That is, this algorithm gener-

ates random networks following the Chung-Lu model. Since in the first step of this algorithm, the edges are picked with equal probability $p$, the edge skipping technique discussed in Sect. 4.3.1 can also be used for this algorithm leading to an $O(m+n)$ time algorithm, which is presented in ${ }^{103}$. A pseudocode for this algorithm using the edge skipping technique is shown in Algorithm 1. whereas $S$ can be computed in parallel in $O\left(\frac{n}{P}+\log P\right)$ time.

- Dividing the task of selecting and generating edges into independent subtasks.

- Balancing computation load among the processors. Load balancing is the most challenging issue in this parallel algorithm.

To compute the sum $S$ in parallel, the weights $W$ are divided equally among $P$ processors such that every processor is responsible for $\frac{n}{P}$ weights. Each of the $P$ processors adds its weights locally in $\frac{n}{P}$ time. Then these local sums from all processors can be aggregated (say, for example, using an MPI reduce function) in $O(\log P)$ time. Therefore, computing sum $S$ takes $O(n / P+\log P)$ time. As

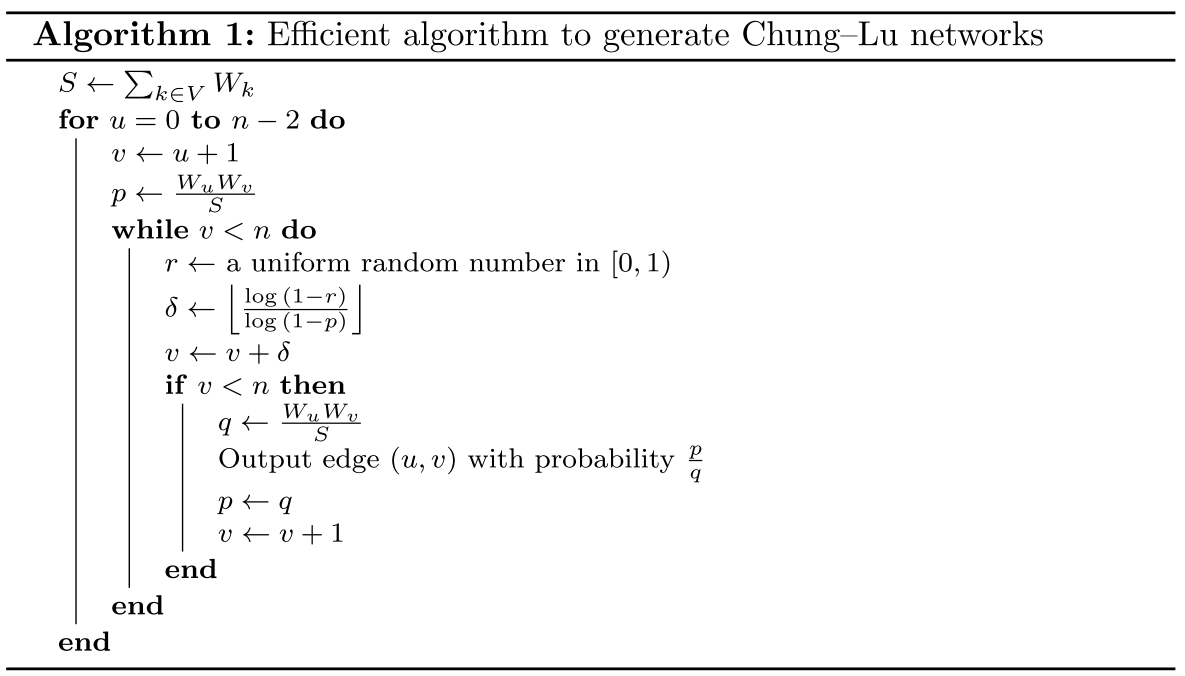

In Algorithm 1, as we always have $u<v$ and no edge $(u, v)$ can be selected more than once, this algorithm does not create any self-loop or parallel edges.

Based on this sequential algorithm, an efficient distributed-memory parallel algorithm is given in $^{5}$ that takes $O\left(\frac{m+n}{P}+P\right)$ time with high probability, where $P$ is the number of parallel processors. Let there be $P$ independent processors with distributed memory system and the processors communicate with each other via exchanging messages. Computation of the probabilities $p_{u, v}$ are dependent on $W_{u}$ and $W_{v}$. Assume that every processor has a copy of the sorted (in nonincreasing order) sequence of the weights in its own memory. Efficient parallelization of Algorithm 1 requires

- Computing the sum $S=\sum_{k=0}^{n-1} W_{k}$ in parallel.

Sequential computation of $S$ takes $O(n)$ time the edges can be generated independently, the iterations of the for loop in Algorithm 1 can be executed by multiple processors independently and simultaneously. For the details of this algorithm along with a good and efficient load balancing method, $\mathrm{see}^{5}$.

\subsection{Epidemiological Simulation}

The GDS formalism of Sect. 4.1 is useful for developing simulation systems ${ }^{90}$. In this section, we look in depth at a simulation system based on the conceptual view of interactions as presented in Sect. 4.1.3. Other tools are cited in Sect. 1.

The EpiSimdemics model ${ }^{26,41,132}$ is used to explore the impact of agent behavior and public policy mitigation strategies on the spread of contagion over extremely large interaction networks. The interaction network is represented by a labeled bipartite graph, where nodes consist of people and locations, referred to as a 


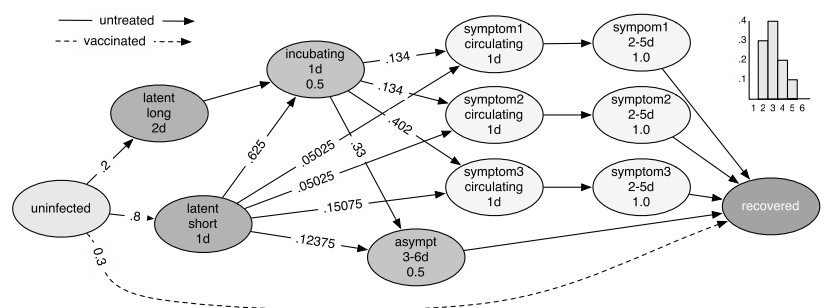

Figure 8: PTTS for the H5N1 disease model. Ovals represent disease states, while lines represent the transition between states, labeled with the transition probabilities. The line type represents the treatment applied to an individual. The states contain a label and the dwell time within the state, and the infectivity if different from one.

person-location graph. If a person visits a location, there is an edge between them, labeled by the type of activity and the time of the visit. The interaction graph is not static, but changes over time in response to changes in a person's health state (e.g., stay home when sick), public policy (e.g., school closure), or behavior changes (e.g., reduce unnecessary activities during an outbreak). This new network, in turn, affects a person's health (e.g., reducing contact with potentially infectious individuals outside the home, or increasing contact with potentially infected children inside the home). Including this co-evolution is important for correctly modeling the spread of disease ${ }^{27}$. The person-location graph is converted to a person-person graph, where nodes represent people and edges represent contact between people, labeled by the duration of contact. This graph is regenerated each timestep as the person-location graph changes.

Between-host contagion transmission and within-host contagion progression can be viewed as two connected but independently computed processes. Between-host transmission triggers the start of within-host progression by causing an uninfected individual to transition to an infected state. The disease progress of the infected individual is then fully determined by the local (vertex) function governing the within-host progression. The within-host disease progression is modeled as a Probabilistic Timed Transition Systems (PTTS), an extension of finite state machines with two additional features: the state transitions are probabilistic and timed. The system also supports multiple interacting PTTSs for modeling of multiple co-circulating diseases, enhanced sociological modeling in the agents, and the addition of more complex interventions, such as contact tracing and antiviral stockpiles.

The PTTS and the interaction network are co-evolving, as the progression of each one potentially affects the other. In simple terms, who you meet determines whether you fall sick, and the progression of a disease may change who you meet (e.g., you stay home because you are sick). The co-evolution can be much more complex, as an individual's schedule may change depending on information exchanged with others, the health state of people they contact even if no infection takes place (e.g., more people than usual are sick at work), or even expected contacts that do not happen (e.g., coworkers who are absent from work). All of this may also be affected by an individual's demographics (e.g., a person's income affects their decision to stay home from work).

\subsubsection{The Disease Model}

The disease propagation (inter-host) and disease progression (intra-host) models were developed in the National Institutes of Health Models of Infectious Disease Agent Study (MIDAS) project. A disease progression model is shown in Fig. 8.

When a susceptible individual and an infectious individual are colocated, the propagation of disease from the infected individual to the susceptible individual is modeled by

$$
p_{i \rightarrow j}=1-\left(1-r_{i} s_{j} \rho\right)^{\tau}
$$

where $p_{i \rightarrow j}$ is the probability of infectious individual $i$ infecting susceptible individual $j, \tau$ is the duration of exposure, $r_{i}$ is the infectivity of $i, s_{j}$ is the susceptibility of $j$, and $\rho$ is the transmissibility, a disease-specific property defined as the probability of a single completely susceptible person being infected by a single completely infectious person during one minute of exposure $^{23}$. Generally, $\rho$ is calibrated to produce a desired attack rate (fraction of total population infected) in the absence of any interventions. A person's infectivity and susceptibility default to 1 , but can be increased or decreased due to 


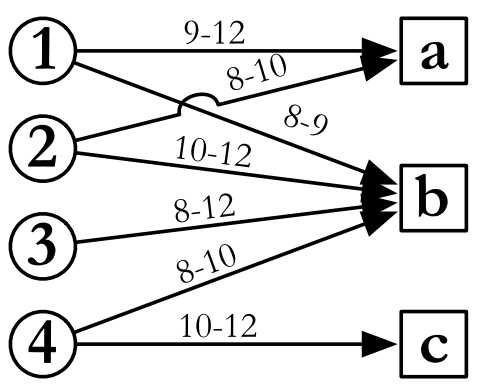

(a) Person-Location Graph

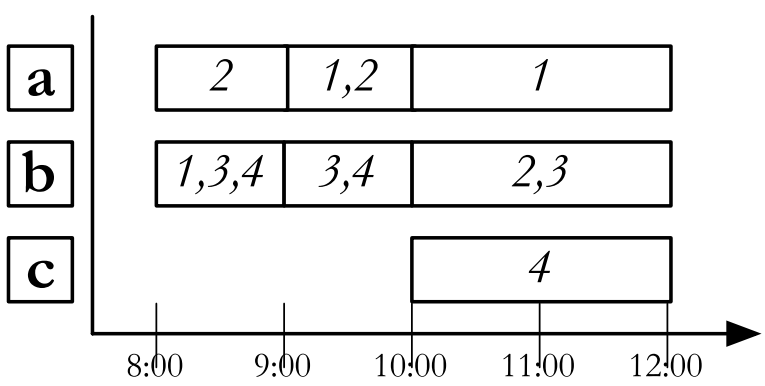

(b) Co-location

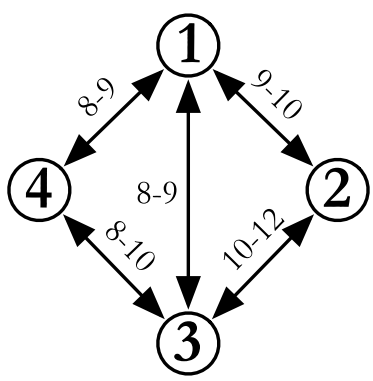

(c) Person-Person Graph

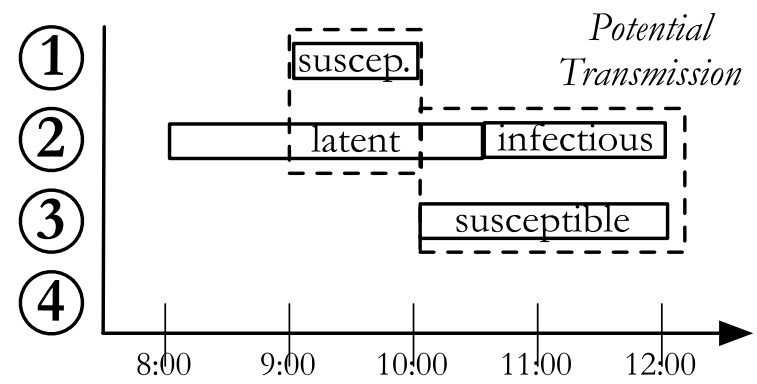

(d) Transmission at location $b$

Figure 9: An example social contact network: (a) the bipartite graph representation showing people visiting locations; (b) the temporal and spatial projection of the network; (c) the person-person graph showing interactions between temporally and spatially co-located people; (d) potential disease transmission between an infectious person 2, and susceptible people 1 and 3 .

individual characteristics or behavioral changes. For instance, a child with less developed personal hygiene habits may be more infectious than typical (i.e., have a infectivity greater than 1 ), while an imuno-compromised individual may have an increased susceptibility. A person who wears a face mask in public may have reduced infectivity and/or susceptibility.

\subsubsection{Intervention and Behavior Modification}

A scenario specifies the behavior of individuals (e.g., stay home when sick), as well as public policy (e.g., school closure when a specific proportion of the students is sick). There are two fundamental changes that can be made that will affect the spread of a contagion in a social network. All behavior and public policy interventions are implemented through these changes. First, the probability of transmission of a contagion can be changed by changing the infectivity or susceptibility of one or more individuals. For example, getting vaccinated reduces an individual's susceptibility whereas wearing a mask while sick reduces an individual's infectivity. Taking antiviral medication, such as TamiFlu (oseltamivir), reduces the likelihood of becoming infected and reduces both the infectivity and length of the infectious period once an infection has taken place. Second, edges can be added, removed, or altered in the social network, resulting in different individuals coming into contact for different amounts of time. Individual behaviors and public policy interventions in EpiSimdemics, collectively referred to as the scenario, expose these two changes in a way that is flexible, easy to understand for the modeler, and computationally efficient.

The scenario is a series of triggers and actions written in a domain specific language. While conceptually simple, this language has proven to be quite powerful in describing a large range of interventions and public policies. A trigger is a conditional statement that is applied to each individual separately. If a trigger evaluates to true, one or more specified actions are executed. These actions can modify an individual by changing its attributes or schedule type, explicitly changing the PTTS and modifying scenario variables. Scenario variables can be written (assigned, incremented, and decremented) and read in the scenario file. The value read is always the value at the end of the previous simulation day. Any writes to a scenario variable are accumulated locally, and synchronized among processors at the end of each simulated day. 


\subsubsection{Social Network Representation}

We provide four views into the simulation system, in terms of populations in Fig. 9. The two networks were addressed previously, but we now include attributes of graph elements that are particular to simulation, and how a simulation uses each type of network to compute contagion dynamics. In EpiSimdemics, the social network is represented by a labeled bipartite graph per Fig. 9a, as discussed previously. Labels attached to persons correspond to his/her demographic attributes such as age or income. The labels attached to locations specify the location's attributes such as its geographic coordinates, the types of activity performed there, maximum capacity, etc. It is important to note that there can be multiple edges between a person and a location which record different visits. Internally, within the EpiSimdemics code, this network is converted into the equivalent person-person graph per Fig. 9c, as discussed earlier, within each (location, sublocation) pair. This form of the contact network is much more conducive for calculating interactions between people, but much less sparse, containing approximately 10 times more edges than the person-location graph. Figure $9 \mathrm{~b}$ shows the people that are colocated in space and time. Assuming that person 2 is infected and either in the latent state (infectious, but not yet symptomatic) or infectious (contagious and symptomatic), Fig. 9d shows a potential transmission. The social contact graph is not static, but changes over time in response to changes in a person's health state (e.g., stay home when sick), public policy (e.g., school closure), or behavior changes (e.g., reduce unnecessary activities during an outbreak). This new network, in turn, affects a person's health (e.g., reducing contact with potentially infectious individuals outside the home, or increasing contact with potentially infected children inside the home). Including this co-evolution is important for correctly modeling the spread of disease ${ }^{27}$.

The EpiSimdemics model can be simulated with a simple discrete event simulation (DES) algorithm in which the system only changes its state upon the occurrence of an event. As shown in Fig. 9d, there are two types of events in the system: Arrive Events (person $p$ arrives at location $l$ at time $t_{\text {arrive }}$ ) and Depart Events (person $p$ leaves location $l$ at time $t_{\text {depart }}$ ).

To ensure correctness, the algorithm has to adhere to the following causality constraint: If an individual $i$ leaves location $L_{A}$ at time $t_{\text {depart }}$ and arrives at location $L_{B}$ at time $t_{\text {arrive }}$, his/her health state when arriving at $L_{B}$ (denoted by $\left.s_{i}\left(t_{\text {arrive }}\right)\right)$ has to be decided prior to calculating the states of other individuals at $L_{B}$ after time $t_{\text {arrive. This }}$ causality constraint leads to temporal and spatial dependencies among nodes in the simulated system.

For simplicity of exposition, travel between locations is shown as instantaneous. In the actual system, there is a delay between leaving one location and arriving at the next location, based on an approximation of the travel time between locations. This delay can be calculated with varying degrees of accuracy ${ }^{12}$.

There are three important semantic points of the contagion diffusion problem that lead to the EpiSimdemics algorithm.

1. Individuals can only affect other individuals through interactions that occur when they are co-located in space and time.

2. An individual's health state changes, once infected, can be precomputed.

3. There is a minimum latent period, $D_{\min }$. This is the amount of time that must pass between a person becoming infected, and a person being able to infect others. For most infectious diseases, there is a suitable latent period that is determined by the biology of the infection. For influenza, this period is at least 24 hours.

The above observations led to a semantics-oriented problem decomposition. The existence of a latent period for newly infected individuals in the disease model provides a basis for relaxing the global clock. If the time period to be simulated is divided into $n$ iterations, and if the length of $a$ single simulation iteration is less than $D_{\text {min }}$, then all locations can be concurrently considered and interactions between individuals at these locations can be simulated in parallel.

The processing is separated into iterations that represent, for influenza, simulated days. It is important to note that state changes are not limited to time step boundaries. For example, if an individual is infected at 10:47 on day 10, and becomes infectious 36 hours later, they can start infecting others at 22:47 on day 11. Each iteration has the basic following four steps.

1. Each individual determines the locations that they are going to visit, based on a normative schedule, public policy, and individual behavior and health state. The person sends a message to each visited location with the details of the visit (time, duration 


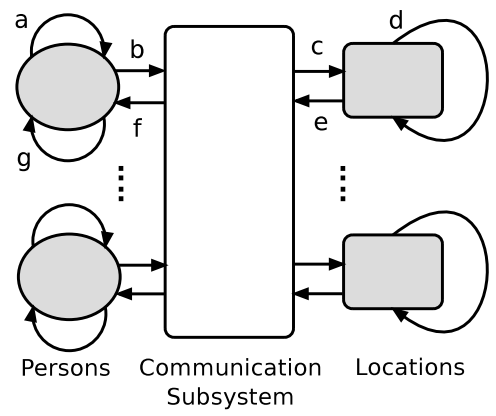

\begin{tabular}{cl}
\hline \multicolumn{2}{c}{ Sequence of operations } \\
\hline \hline a: & person computes visits \\
b: & person sends visits \\
c: & location receives visits \\
d: & location computes visits \\
e: & location sends outcomes \\
f: & person receives outcomes \\
g: & person combines outcomes \\
\hline
\end{tabular}

Figure 10: The computational structure of the sequential EpiSimdemics algorithm.

and health state during the visit). This can be computed in parallel for each person.

2. Each location computes the pairwise interactions that occur between occupants of that location. Each interaction may or may not result in an infection, depending on a stochastic model. For the epidemiological model, disease propagation is modeled by Equation 4.

A message is then sent to each infected person with the details of the infection (time of infection, infector and location). Again, each location can perform this computation in parallel, once it has received information for all of the individuals that will visit the location during the iteration.

3. Each person who is infected updates its health state by transitioning to an infected state. In the event that a person iis infected in multiple locations, the earliest infection is chosen.

4. Any global simulation states (i.e., total people infected) are updated.

For each iteration, there are two synchronizations required: between steps 1 and 2, and between steps 2 and 3 . In addition, step 4 requires a reduction operation. These computational steps are further broken down in Fig. 10.

\subsubsection{Performance}

EpiSimdemics has been carefully designed to balance generality, efficiency, and scalability. It has been used to simulate the United States population (on the order of 300 million people) on both moderate sized university clusters of 1000 cores and NCSA's BlueWaters system of 352,000 cores. The latter system was able to simulate the spread for 120 simulated days in less than 12 seconds $^{133}$, as shown in Fig. 11.

\section{Policy Implications}

One of the most important practical results of epidemic simulations is to inform policy planning. A listing of selected studies is provide in Table 2. A few of these studies are described below. We note that simulation is not the only way to generate results to inform policy. For example, such results can be generated with compartmental models ${ }^{101}$ and game theoretic approaches $^{34}$, which are not covered here.

Reference $^{73}$ describes a multi-institutional study, exercising three different ABMs, to determine the most effective strategies for mitigating influenza spread in a 9 million agent system, similar to a population like Chicago. They found that a combination of school closures and targeted antiviral prophylaxis by individuals gave good results in decreasing the number of infected people. Additional work ${ }^{41}$ indicates that these results are robust across a different population.

In taking the ${ }^{73}$ study one step further, ${ }^{15}$ looked not only at outbreak size, but also the costs of outbreaks to determine which intervention strategies were most cost-effective. These costs include lost productivity by corporations, as well as lost income by households. So, for example, while staying at home (i.e., social distancing) may be useful in halting transmission, it also has the cost of reducing income among some socio-economic classes. Of the strategies investigated, the one that reduces the size of the outbreak and total costs is a combination of behavior modification of individuals (i.e., eliminating non-essential travel and taking antivirals) and government action (i.e., closing schools). In this case, the number of infected individuals decreases by $87 \%$ and the total cost drops by $82 \%$. These findings indicate that the strategies that are best for decreasing outbreak size are also good for reducing their economic impact. Furthermore, it is noted in ${ }^{99}$ that paid sick leave is also cost-effective because it reduces 


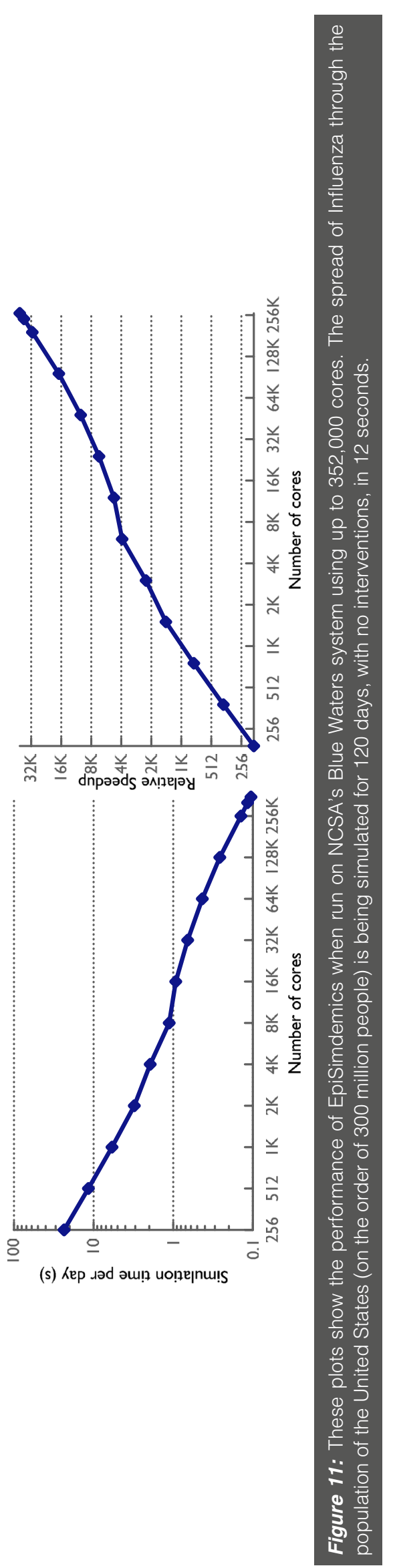


Table 2: Selected studies pertaining to epidemic simulations to support policy planning. The great majority of these investigate intervention strategies to reduce disease outbreaks.

\begin{tabular}{llll}
\hline Number & Type of study & Description & References \\
\hline 1 & Epidemiological & $\begin{array}{c}\text { Determine which intervention strategies are most effective in } \\
\text { reducing outbreak size } \\
\text { Determine which intervention strategies are most cost-effective; } \\
\text { i.e., the reduction in outbreak size per unit expenditure }\end{array}$ & 73 \\
\hline 2 & Economic & $\begin{array}{l}\text { Different intervention triggers by demographic class } \\
\text { Simulations run during a large outbreak by policy planners for situ- }\end{array}$ & 36 \\
\hline 4 & Epidemic tracking & $\begin{array}{l}\text { Stional awareness } \\
\text { Demonstrates, for stylized networks, that homogeneous vaccina- }\end{array}$ & 134 \\
\hline 5 & Epidemiological & $\begin{array}{l}\text { tion strategies can be counterproductive and that strategies } \\
\text { should depend on social network local conditions }\end{array}$ & 98 \\
\hline 6 & Epidemiological & $\begin{array}{c}\text { Comparisons of vaccination strategies based on local versus } \\
\text { regional conditions }\end{array}$ & 1 \\
\hline 7 & Epidemiological & Evaluation of drugs and vaccines that are under development & 32 \\
\hline 8 & Epidemiological & Influenza-based interventions for school-age children & 88 \\
\hline 10 & Economic & Eaid sick leave and its effect on outbreak size & 62 \\
\hline 11 & Epidemiological & Influenza outbreak and various containment strategies & 100 \\
\hline 12 & Epidemiological and social & Interventions for influenza in (slums of) Delhi, India & 3 \\
\hline
\end{tabular}

the spread of sick workers who would otherwise come to work. Paid sick leave is also studied elsewhere (e.g., ${ }^{88}$ ).

\section{Summary}

In this paper, we motivated epidemic simulation and itemized challenges in developing capabilities to perform these simulations. We focused primarily on describing fundamental elements of epidemic simulation: (i) theoretical foundations for simulation software, (ii) synthetic population (digital twin) development, (iii) social networks generated from synthetic populations, (iv) large-scale stylized network generation, and (v) simulation. We provided several examples of how epidemic simulation can support policy planning.

\section{Publisher's Note}

Springer Nature remains neutral with regard to jurisdictional claims in published maps and institutional affiliations.

\section{Acknowledgements}

We thank the reviewers for their detailed comments for improving the paper. This work is partially supported by NSF Grants CMMI-1916670
(CRISP 2.0), ACI-1443054 (DIBBS), IIS-1633028 (BIG DATA), IIS-1931628 (BIG DATA), CMMI1745207 (EAGER), OAC-1916805 (CINES), and CCF-1918656 (Expeditions).

Received: 2 December 2020 Accepted: 2 June 2021

Published online: 5 October 2021

\section{References}

1. Abu-Raddad LJ, Sabatelli L, Achterberg JT, Sugimoto JD, Longini Ira MJ, Dyee C, Halloran ME (2009) Epidemiological benefits of more-effective tuberculosis vaccines, drugs, and diagnostics. Proc Natl Acad Sci 106:13980-13985

2. Adiga A, Kuhlman CJ, Mortveit HS, Wu S (2015) Effect of graph structure on the limit sets of threshold dynamical systems. In: Proc. Cellular Automata and Discrete Complex Systems - 21st IFIP WG 1.5 International Workshop, AUTOMATA 2015, Turku, Finland, June 8-10, 2015., pp 59-70

3. Adiga A, Chu S, Eubank S, Kuhlman CJ, Lewis B, Marathe A, Nordberg E, Swarup S, Vullikanti A, Wilson ML (2018) Disparities in spread and control of influenza in slums of Delhi: Findings from an agent-based modeling study. BMJ Open 2:2

4. Adiga A, Kuhlman CJ, Marathe MV, Mortveit HS, Ravi SS, Vullikanti A (2019) Graphical dynamical systems and their applications to bio-social systems. Int J Adv Eng Sci Appl Math 11:153-171 
5. Alam M, Khan M (2017) Parallel algorithms for generating random networks with given degree sequences. Int J Parallel Prog 45:109-127

6. Alam M, Khan M, Marathe MV (2013) Distributedmemory parallel algorithms for generating massive scale-free networks using preferential attachment model. In: Proceedings of the Intl. Conf. for High Performance Computing, Networking, Storage and Analysis (SuperComputing)

7. Alam M, Khan M, Vullikanti A, Marathe M (2016) An efficient and scalable algorithmic method for generating large-scale random graphs. In: SC '16: Proceedings of the International Conference for High Performance Computing, Networking, Storage and Analysis, pp 372-383, https://doi.org/10.1109/SC.2016.31

8. Alam M, Khan M, Perumalla KS, Marathe M (2020) Generating massive scale-free networks: Novel parallel algorithms using the preferential attachment model. ACM Transactions on Parallel Computing $7(2)$

9. Albert R, Jeong H, Barabási AL (2000) Error and attack tolerance of complex networks. Nature 406(6794):378382. https://doi.org/10.1038/35019019

10. Arentze T, Timmermans H, Hofman F (2014) Creating synthetic household populations: problem and approach. J Transport Res Board 2:85-91

11. Barabási AL, Albert R (1999) Emergence of scaling in random networks. Science (New York, NY) 286(5439):509-512 http://view.ncbi.nlm.nih.gov/pubmed/10521342

12. Barrett C, Beckman R, Berkbigler K, Bisset K, Bush B, Campbell K, Eubank S, Henson K, Hurford J, Kubicek D, Marathe M, Romero P, Smith J, Smith L, Speckman P, Stretz P, Thayer G, Eeckhout E, Williams MD (2001) TRANSIMS: Transportation analysis simulation system. Tech. Rep. LA-UR-00-1725. An earlier version appears as a 7 part technical report series LA-UR-99-1658 and LA-UR-99-2574 to LA-UR-99-2580, Los Alamos National Laboratory Unclassified Report

13. Barrett C, Beckman R, Khan M, Kumar VSA, Marathe M, Stretz P, Dutta T, Lewis B (2009) Generation and analysis of large synthetic social contact networks. In: Winter Simulation Conference (WSC)

14. Barrett C, Bisset K, Leidig J, Marathe A, Marathe MV (2010) An integrated modeling environment to study the coevolution of networks, individual behavior and epidemics. AI Mag 31:75-87

15. Barrett C, Bisset K, Leidig J, Marathe A, Marathe M (2011) Economic and social impact of influenza mitigation strategies by demographic class. Epidemics 3:19-31

16. Barrett C, Eubank S, Marathe A, Marathe M, Pan Z, Swarup S (2011) Information integration to support policy informatics. Innov J 2:1-19

17. Barrett CL, Hunt III HB, Marathe MV, Ravi SS, Rosenkrantz DJ, Stearns RE (2001) Analysis problems for sequential dynamical systems and communicating state machines. In: MFCS, pp 159-172

18. Barrett CL, Hunt III HB, Marathe MV, Ravi SS, Rosenkrantz DJ, Stearns RE, Tosic PT (2001) Gardens of Eden and fixed points in sequential dynamical systems. In: DM-CCG, pp 95-110

19. Barrett CL, Hunt HB III, Marathe MV, Ravi SS, Rosenkrantz DJ, Stearns RE (2003) On special classes of sequential dynamical systems. Ann Comb 7:381-408

20. Barrett CL, Hunt III HB, Marathe MV, Ravi SS, Rosenkrantz DJ, Stearns RE (2003) Predecessor and permutation existence problems for sequential dynamical systems. In: DMCS, pp 69-80

21. Barrett CL, Hunt HB III, Marathe MV, Ravi SS, Rosenkrantz DJ, Stearns RE (2003) Reachability problems for sequential dynamical systems with threshold functions. Theoret Comput Sci 295:41-64

22. Barrett CL, Hunt HB III, Marathe MV, Ravi SS, Rosenkrantz DJ, Stearns RE (2006) Complexity of reachability problems for finite discrete dynamical systems. J Comput Syst Sci 72(8):1317-1345

23. Barrett CL, Bisset K, Eubank S, Marathe MV, Kumar VA, Mortveit H (2007) Modeling and Simulation of Biological Networks, AMS, chap Modeling and Simulation of Large Biological. An Interaction Based Approach, Information and Socio-Technical Systems, pp 101-147

24. Barrett CL, Hunt HB III, Marathe MV, Ravi SS, Rosenkrantz DJ, Stearns RE, Thakur M (2007) Predecessor existence problems for finite discrete dynamical systems. Theoret Comput Sci 386(1-2):3-37

25. Barrett CL, III HBH, Marathe MV, Ravi SS, Rosenkrantz DJ, Stearns RE, Thakur M (2007) Computational aspects of analyzing social network dynamics. In: IJCAI 2007, Prc. 20th International Joint Conference on Artificial Intelligence, Hyderabad, India, January 6-12, 2007, pp 2268-2273

26. Barrett CL, Bisset KR, Eubank SG, Feng X, Marathe MV (2008) Episimdemics: an efficient algorithm for simulating the spread of infectious disease over large realistic social networks. In: Proceedings of the 2008 ACM/IEEE conference on Supercomputing, IEEE Press, Piscataway, NJ, USA, SC '08, pp 37:1-37:12, http://dl.acm.org/citat ion.cfm?id=1413370.1413408

27. Barrett CL, Eubank S, Marathe MV (2008) An interaction based approach to computational epidemics. In: AAAI' 08: Proceedings of the Annual Conference of AAAI, AAAI Press, Chicago USA

28. Barrett CL, Hunt HB III, Marathe MV, Ravi SS, Rosenkrantz DJ, Stearns RE (2011) Modeling and analyzing social network dynamics using stochastic discrete graphical dynamical systems. Theoret Comput Sci 412(30):3932-3946

29. Barthelemy J, Cornelis E (2012) Synthetic populations: review of the different approaches 
30. Barthelemy J, Toint PL (2011) Synthetic population generation without a sample. Inf Transport Sci 47:266-279

31. Bartik AW, Bertrand M, Cullen Z, Glaeser EL, Luca M, Stanton C (2020) The impact of covid-19 on small business outcomes and expectations. Proc Natl Acad Sci 117(30):17656-17666

32. Basta NE, Chao DL, Halloran ME, Matrajt L, Longini Ira MJ (2009) Strategies for pandemic and seasonal influenza vaccination of schoolchildren in the united states. Am J Epidemiol 170:679-686

33. Batagelj V, Brandes U (2005) Efficient generation of large random networks. Phys Rev E 71(3):36113. https://doi.org/10.1103/PhysRevE.71.036113

34. Bauch CT, Earn DJD (2004) Vaccination and the theory of games. Proc Natl Acad Sci 101(36):13391-13394

35. Beckman R, Channakeshava K, Huang F, Kim J, Marathe A, Marathe M, Pei G, Saha S, Vullikanti AKS (2013) Integrated multi-network modeling environment for spectrum management. IEEE J Sel Areas Commun 31(6):1158-1168

36. Beckman R, Bisset KR, Chen J, Lewis B, Marathe M, Stretz P (2014) Isis: A networked-epidemiology based pervasive web app for infectious disease pandemic planning and response. In: Proceedings of the 20th ACM SIGKDD International Conference on Knowledge Discovery and Data Mining, ACM, New York, NY, USA, KDD '14, pp 1847-1856, https://doi.org/10.1145/26233 30.2623375

37. Beckman RJ, Baggerly KA, McKay MD (1996) Creating synthetic base-line populations. Transport Res A Policy Pract 30:415-429

38. Bhatele A, Yeom JS, Jain N, Kuhlman C, Livant Y, Bisset K, Kale LV, Marathe M (2017) Massively parallel simulations of spread of infectious diseases over realistic social networks. In: ACM/IEEE International Symposium on Cluster, Cloud, and Grid Computing (CCGRID), pp 689-694

39. Bisset K, Marathe M (2009) A cyber-environment to support pandemic planning and response. DOE SciDAC Magazine 36-47

40. Bisset KR, Chen J, Feng X, Kumar VSA, Marathe MV (2009) EpiFast: a fast algorithm for large scale realistic epidemic simulations on distributed memory systems. In: Proceedings of the 23rd International Conference on Supercomputing, pp 430-439

41. Bisset KR, Feng X, Marathe M (2009) Modeling interaction between individuals, social networks and public policy to support public health epidemiology. In: Winter Simulation Conference (WSC)

42. Bjornstad ON, Shea K, Krzywinski M, Altman N (2020) Modeling infectious epidemics. Nat Methods 17:453-456

43. Bonds MH, Keenan DC, Rohani P, Sachs JD (2009) Poverty trap formed by the ecology of infectious disease. Proc R Soc B 277:1185-1192
44. von Braun J, Zamagni S, Sorondo MS (2020) The moment to see the poor. Science 368:214

45. Centers for Disease Control and Prevention (2015) Ebola (ebola virus disease). http://www.cdc.gov/vhf/ ebola/outbreaks/2014-west-africa/, http://www.cdc. gov/vhf/ebola/outbreaks/2014-west-africa/ (Visited 30 January 2015)

46. Centers for Disease Control and Prevention (2015) Influenza (flu). http://www.cdc.gov/flu/about/qa/ disease.htm, visited 30 January 2015

47. Centers for Disease Control and Prevention (2015) Severe acute respiratory syndrome (sars). http://www. cdc.gov/sars/about/fs-SARS.html, http://www.cdc. gov/sars/about/fs-SARS.html (Visited 24 February 2015)

48. Centola D, Macy M (2007) Complex contagions and the weakness of long ties1. Am J Sociol 113(3):702-734

49. Chao DL, Halloran ME, Obenchain VJ Jr, IML, (2010) FluTE, a publicly available stochastic influenza epidemic simulation model. PLoS Comput Biol 6:e1000656

50. Chapuis K, Taillandier P (2019) A brief review of synthetic population generation practices in agent-based social simulation. In: Social Simulation for Policy; SP2S: Synthetic population in social simulation

51. Choupani AA, Mamdoohi AR (2016) Population synthesis using iterative proportional fitting (ipf): A review and future research. Transportation Research Procedia 223-233

52. Chung F, Lu L (2002) Connected components in random graphs with given expected degree sequences. Ann Comb 6(2):125-145. https://doi.org/10.1007/PL000 12580

53. Courtney D, Watson P, Battaglia M, Mulsant BH, Szatmari P (2020) Covid-19 impacts on child and youth anxiety and depression: Challenges and opportunities. Can J Psychiatry 65(10):688-691

54. Cutts FT, Dansereau E, Ferrari MJ, Hanson M, McCarthy KA, Metcalf CJE, Takahashi S, Tatem AJ, Thakkar N, Truelove S, Utazi E, Wesolowski A, Winter AK (2020) Using models to shape measles control and elimination strategies in low- and middle-income countries: A review of recent applications. Vaccine 38:979-992

55. Deming WE, Stephan FF (1940) On a least squares adjustment of a sampled frequency table when the expected marginal tables are known. Ann Math Stats 11(4):427-444

56. Epstein J (2007) Generative Social Science: Studies in Agent-Based Computational Modeling. Princeton University Press

57. Epstein JM, Parker J, Cummings D, Hammond RA (2008) Coupled contagion dynamics of fear and disease: mathematical and computational explorations. PLoS ONE 3:3955 
58. Erdös P, Rényi A (1960) On the evolution of random graphs. In: Publications of the Mathematical Institute of the Hungarian Academy of Sciences, pp 17-61

59. Eubank S, Guclu H, Kumar VSA, Marathe M, Srinivasan V, Toroczkai Z, Wan N (2004) Modelling disease outbreaks in realistic urban social networks. Nature 429:180-184

60. Eubank S, Kumar VSA, Marathe M, Srinivasan A, Wang N (2006) Structure of social contact networks and their impact on epidemics. AMS-MIMACS Special Volume on Epidemiology

61. Eubank S, Barrett C, Beckman R, Bisset K, Durbeck L, Kuhlman CJ, Lewis B, Marathe A, Marathe M, Stretz P (2010) Detail in network models of epidemiology: are we there yet? J Biol Dyn 4:446-455

62. Ferguson NM, Cummings DAT, Fraser C, Cajka JC, Cooley PC, Burke DS (2006) Strategies for mitigating an influenza pandemic. Nat Lett 442:448-452

63. Fortuna LR, Tolou-Shams M, Porche MW (2020) Inequity and the disproportionate impact of covid-19 on communities of color in the united states: The need for a trauma-informed social justice response. Psychol Trauma 12(5):443-445

64. Frick M, Axhausen KW (2004) Generating synthetic populations using ipf and monte carlo techniques: Some new results. In: 4th Swiss Transport Research Conference (STRC)

65. Fujimoto RM (2000) Parallel and distributed simulation systems. Wiley-Interscience

66. Funke D, Lamm S, Sanders P, Schulz C, Strash D, von Looz M (2018) Communication-free massively distributed graph generation. In: 2018 IEEE International Parallel and Distributed Processing Symposium (IPDPS), pp 336-347, https://doi.org/10.1109/IPDPS.2018.00043

67. Gilbert N (2007) Agent-based models. Sage Publishing

68. Giordano G, Blanchini F, Bruno R, Colaneri P, Filippo $\mathrm{AD}$, Matteo AD, Colaneri M (2020) Modelling the covid-19 epidemic and implementation of populationwide interventions in italy. Nat Med 26:855-860

69. Goles E, Martinez S (1990) Neural and automata networks. Kluwer Academic Publishers

70. González MC, Hidalgo CA, Barabási AL (2008) Understanding human mobility patterns. Nature 453:779-782

71. Granovetter M (1978) Threshold models of collective behavior. Am J Sociol 83(6):1420-1443

72. Grefenstette JJ, Brown ST, Rosenfeld R, Depasse J, Stone NT, Cooley PC, Wheaton WD, Fyshe A, Galloway DD, Sriram A, Guclu H, Abraham T, Burke DS (2013) Fred (a framework for reconstructing epidemic dynamics): An open-source software system for modeling infectious diseases and control strategies using census-based populations. BMC Public Health $13: 2$

73. Halloran ME, Ferguson NM, Eubank S, Longini IM, Cummings DAT, Lewis B, Xu S, Fraser C, Vullikanti A, Germann TC, Wagener D, Beckman R, Kadau K, Barrett
C, Macken CA, Burke DS, Cooley P (2008) Modeling targeted layered containment of an influenza pandemic in the united states. Proc Natl Acad Sci (PNAS) 105:4639-4644

74. Harland K, Heppenstall A, Smith D, Birkin M (2012) Creating realistic synthetic populations at varying spatial scales: A comparative critique of population synthesis techniques. J Artif Soc Soc Simul 15:2

75. Hethcote HW (2000) The mathematics of infectious diseases. Soc Ind Appl Math 42:599-653

76. Hoertel N, Blachier M, Blanco C, Olfson M, Massetti M, Limosin MSRF, Leleu H (2020) A stochastic agentbased model of the sars-cov-2 epidemic in france. Nat Med 26:1417-1421

77. Ireland CT, Kullback S (1968) Contingency tables with given marginals. Biometrika 55(1):179-188

78. Kempe D, Kleinberg JM, Tardos É (2003) Maximizing the spread of influence through a social network. In: Getoor L, Senator TE, Domingos P, Faloutsos C (eds) KDD, ACM, pp 137-146, https://doi.org/10.1145/ 956750.956769

79. Kilbourne ED (2006) Influenza pandemics of the 20th century. Emerging Infectious Diseases 9-14

80. Kofman YB, Garfin DR (2020) Home is not always a haven: The domestic violence crisis amid the covid-19 pandemic. Psychol Trauma Theory Res Pract Policy 12(S1):S199-S201

81. Kohei T, Naoki M (2017) Effects of the distant population density on spatial patterns of demographic dynamics. R Soc Open Science

82. Kosub S, Homan CM (2007) Dichotomy results for fixed point counting in boolean dynamical systems. In: Proc. ICTCS, pp 163-174

83. Kuhlman CJ, Mortveit HS (2015) Limit sets of generalized, multi-threshold networks. J Cell Autom 10:161-193

84. Kuhlman CJ, Mortveit HS, Murrugarra D, Kumar VSA (2011) Bifurcations in boolean networks. In: AUTOMATA, pp 29-46

85. Kuhlman CJ, Kumar VSA, Marathe MV, Ravi SS, Rosenkrantz DJ (2015) Inhibiting diffusion of complex contagions in social networks: theoretical and experimental results. Data Min Knowl Discov 29(2):423-465

86. Kuhlman CJ, Ren Y, Lewis BL, Schlitt J (2017) Hybrid agent-based modeling of zika in the united states. In: Winter Simulation Conference (WSC), pp 1085-1096

87. Kumar R, Raghavan P, Rajagopalan S, Sivakumar D, Tomkins A, Upfal E (2000) Stochastic models for the web graph. In: Proceedings of the 41st Annual Symposium on Foundations of Computer Science (FOCS), IEEE Computer Society, Washington, DC, USA, p 57, http://dl.acm.org/citation.cfm?id=795666.796570

88. Kumar S, Grefenstette JJ, Galloway D, Albert SM, Burke DS (2013) Policies to reduce influenza in the workplace: Impact assessments using an agent-based model. Am J Public Health 103(8):1406-1411 
89. Kuylen E, Willem L, Broeckhove J, Beutels P, Hens N (2020) Clustering of susceptible individuals within households can drive measles outbreaks: an individualbased model exploration. Sci Rep 10:13

90. Laubenbacher R, Jarrah AS, Mortveit HS, Ravi SS (2020) Mathematical formalism for agent-based modeling. Compl Soc Behav Syst 2:683-703

91. Lenormand M, Deffuant G (2013) Generating a synthetic population of individuals in households: samplefree vs sample-based methods. J Artif Soc Soc Simul $16: 2$

92. Leskovec J (2008) Dynamics of large networks. Pittsburgh, PA, USA, $p$ aAI3340652. PhD thesis

93. Letendre K, Fincher CL, Thornhill R (2010) Does infectious disease cause global variation in the frequency of intrastate armed conflict and civil war? Biol Rev 85:669-683

94. Little RJA, Wu MM (1991) Models for contingency tables with known mmargin when target and sampled populations differ. J Am Stat Assoc 86(413):87-95

95. Lum K, Chungbaek Y, Eubank SG, Marathe MV (2013) A two-stage, fitted values approach to activity matching. In: Procedia - Social and Behavioral Sciences

96. Ma L, Srinivasan S (2015) Synthetic population generation with multilevel controls: a fitness-based synthesis approach and validations. Comput-Aided Civ Infrastruct Eng 30(2):135-150. https://doi.org/10.1111/ mice. 12085

97. Macauley M, Mortveit HS (2009) Cycle equivalence of graph dynamical systems. Nonlinearity 22:421-436

98. Marathe A, Lewis B, Barrett C, Chen J, Marathe M, Eubank S, Ma Y (2011) Comparing effectiveness of topdown and bottom-up strategies in containing influenza. PLoS ONE 6:e25149-1-e25149-6

99. Marathe A, Chen J, Eubank S, Liao S, Ma Y (2014) Impact of paid sick leave policy: a social planners perspective. Am J Public Health 104:1

100. Marathe A, Chen J, Chu S, Chungbaek Y, Khan M, KuhIman C, Mortveit H, Vullikanti A, Xie D (2016) Effect of modeling slum populations on influenza spread Delhi. BMJ Open 2:2

101. Medlock J, Galvani AP (2009) Optimizing influenza vaccine distribution. Science 325(5948):1705-1708

102. Meindl B, Templ M, Alfons A, Kowarik A (2014) simpop : An open source $R$ package for generating synthetic populations. http://www.ihsn.org/home/projects/ synthetic-populations

103. Miller J, Hagberg A (2011) Efficient generation of networks with given expected degrees. In: Proceedings of Algorithms and Models for the Web-Graph (WAW), pp $115-126$

104. Moeckel R, Spiekermann K, Wegener M (2003) Creating a synthetic population. In: 8th International Conference on Computers in Urban Planning and Urban Management (CUPUM)
105. Mortveit HS, Reidys C (2007) An introduction to sequential dynamical systems. Springer

106. Müller K, Axhausen K (2010) Population synthesis for microsimulation: State of the art. Tech. rep., Technical Report August. Swiss Federal Institute of Technology Zurich

107. Muller K, Axhausen KW (2011) Hierarchical ipf: Generating a synthetic population for switzerland. In: ERSA

108. Namazi-Rad MR, Mokhtarian P, Perez P (2014) Generating a dynamic synthetic population-using an agestructured two-sex model for household dynamics. PLoS ONE 9:e4761-1-e4761-16

109. National Center for Education Statistics (2013) Characteristics of public and private elementary and secondary schools in the United States: Results from the 2011d12 schools and staffing survey. Tech. Rep. NCES 2013312, Department of Education

110. National Center for Education Statistics (2014) Private school universe survey (PSS): Public-use data file user's manual for school year 2011-12. Tech. Rep. NCES 2014351, Department of Education

111. Nobari S, Lu X, Karras P, Bressan S (2011) Fast random graph generation. In: Proceedings of the 14th International Conference on Extending Database Technology (EDBT/ICDT), pp 331-342, https://doi.org/10.1145/ 1951365.1951406

112. Railsback SF, Grimm V (2011) Agent-based and individual-based modeling: a practical introduction. Princeton University Press

113. Ramadan OE, Sisiopiku VP (2019) A critical review on population synthesis for activity- and agent-based transportation models

114. Reluga TC, Medlock J, Perelson AS (2008) Backward bifurcations and multiple equilibria in epidemic models with structured immunity. J Theor Biol 252:155-165

115. Rivers CM, Lofgren ET, Marathe MV, Eubank S, Lewis BL (2014) Modeling the impact of interventions on an epidemic of ebola in sierra leone and liberia. PLOS Curr Outbreaks

116. Rosenkrantz DJ, Marathe MV, Ravi SS, Stearns RE (2018) Testing phase space properties of synchronous dynamical systems with nested canalyzing local functions. In: Autonomous Agents and Multi-Agent Systems (AAMAS), pp 1585-1594

117. Rosenthal DM, Ucci M, Heys M, Hayward A, Lakhanpaul M (2020) Impacts of covid-19 on vulnerable children in temporary accommodation in the uk. Lancet Public Health 5:E241-E242

118. Santos A, McGuckin N, Nakamoto H, Gray D, Liss S (2011) Summary of travel trends: 2009 National Household Travel Survey. Tech. Rep. FHW A-PL-11-022, U.S. Department of Transportation Federal Highway Administration

119. Schelling $\mathrm{T}$ (1978) Micromotives and macrobehavior. Norton and Co., New York 
120. Shrira I, Wisman A, Webster GD (2013) Guns, germs, and stealing: Exploring the link between infectious disease and crime. Evol Psychol 11:270-287

121. Stearns RE, Ravi SS, Marathe MV, Rosenkrantz DJ (2019) Symmetry properties of nested canalyzing functions. In: Discrete Mathematics \& Theoretical Computer Science, $\mathrm{p} 17$ pages

122. Tosic PT (2010) On the complexity of enumerating possible dynamics of sparsely connected boolean network automata with simple update rules. In: Automata 2010 - 16th Intl. Workshop on CA and DCS, pp $125-144$

123. Truscott J, Ferguson NM (2012) Evaluating the adequacy of gravity models as a description of human mobility for epidemic modelling. PLoS Comput Biol 8:1-12

124. Usher K, Bhullar N, Durkin J, Gyamfi N, Jackson D (2020) Family violence and covid-19: Increased vulnerability and reduced options for support. Int J Ment Health Nurs 29:549-552

125. Vigo D, Patten S, Pajer K, Krausz M, Taylor S, Rush B, Raviola G, Saxena S, Thornicroft G, Yatham LN (2020) Mental health of communities during the covid-19 pandemic. Can J Psychiatry 65(10):681-687

126. WebMD (2015) Cold, flu, \& cough health center. http:// www.webmd.com/cold-and-flu/what-are-epidemicspandemics-outbreaks, visited 30 January 2015

127. Wu S, Adiga A, Mortveit HS (2014) Limit cycle structure for dynamic bi-threshold systems. Theoret Comput Sci 559:34-41

128. Xia H, Barrett CL, Chen J, Marathe MV (2013) Computational methods for testing adequacy and quality of massive synthetic proximity social networks. In: Proc. IEEE International Conference on Big Data Science and Engineering (BDSE)

129. Xia H, Chen J, Marathe MV, Swarup S (2014) Comparison and validation of synthetic social contact networks for epidemic modeling (extended abstract). In: Proceedings of The Thirteenth International Conference on Autonomous Agents and Multiagent Systems (AAMAS), Paris, France

130. Yaméogo BF, Gastineau P, Hankach P, Vandanjon PO (2020) Comparing methods for generating a twolayered synthetic population. Transportation Research Record 223-233

131. Ye X, Konduri K, Pendyala RM, Sana B, Waddell P (2009) A methodology to match distributions of both household and person attributes in the generation of synthetic populations. http://urbanmodel.asu.edu/ popgen/papers/PopulationSynthesizerPaper_TRB.pdf, submitted for Presentation Only to the 88th Annual Meeting of the Transportation Research Board, January 11-15, 2009

132. Yeom JS, Bhatele A, Bisset K, Bohm E, Gupta A, Kale L, Marathe M, Nikolopoulos D, Schulz M, Wesolowski L (2014) Overcoming the scalability challenges of epidemic simulations on Blue Waters. In: Parallel and Distributed Processing Symposium, 2014 IEEE 28th International, pp 755-764, https://doi.org/10.1109/IPDPS. 2014.83

133. Yeom JS, Bhatele A, Bisset KR, Bohm E, Gupta A, Kale LV, Marathe M, Nikolopoulos DS, Schulz M, Wesolowski L (2014) Overcoming the scalability challenges of epidemic simulations on Blue Waters. In: 28th IEEE International Parallel \& Distributed Processing Symposium (IPDPS)

134. Yi M, Marathe A (2013) Policy trap and optimal subsidization policy under limited supply of vaccines. PLoS ONE 8:e67249-1-e67249-9

135. Zhu Y, Joseph Ferreira J (2014) Synthetic population generation at disaggregated spatial scales for land use and transportation microsimulation. http://assets.confe rencespot.org/fileserver/file/66219/filename/14-5313. pdf, transportation Research Board Annual Meeting

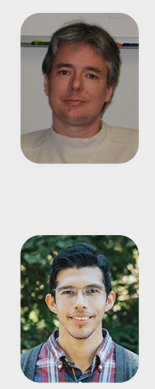

Keith R. Bissett is a Senior Software Engineer at Apple. His research interests include high performance computing, cloud services, and software systems architectures.

Jose Cadena is a Research Staff Member in the Computational Engineering Division at Lawrence Livermore National Laboratory. His research interests include graph mining, machine learning for network data, combinatorial optimization, and approximation

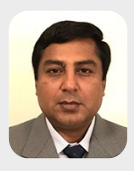

Maleq Khan is an Assistant Professor in the Department of Electrical Engineering and Computer Science at Texas A\&M University-Kingsville. His research interests include parallel and distributed algorithms, high performance computing, graphs analytics, data mining, and computational epidemiology.

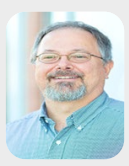

Chris J. Kuhlman is a Research Associate Professor in the Biocomplexity Institute at the University of Virginia. His research interests include modeling of human behavior, simulation, high performance and distributed computing, algorithms, and net-

work science. 